\title{
Revealing Hidden Structural Order Controlling Both Fast and Slow Glassy Dynamics in Supercooled Liquids
}

\author{
Hua Tong and Hajime Tanaka* \\ Department of Fundamental Engineering, Institute of Industrial Science, University of Tokyo, \\ 4-6-1 Komaba, Meguro-ku, Tokyo 153-8505, Japan
}

(Received 21 September 2017; revised manuscript received 10 January 2018; published 14 March 2018)

The dynamics of a supercooled liquid near the glass transition is characterized by two-step relaxation, fast $\beta$ and slow $\alpha$ relaxations. Because of the apparently disordered nature of glassy structures, there have been long debates over whether the origin of drastic slowing-down of the $\alpha$ relaxation accompanied by heterogeneous dynamics is thermodynamic or dynamic. Furthermore, it has been elusive whether there is any deep connection between fast $\beta$ and slow $\alpha$ modes. To settle these issues, here we introduce a set of new structural order parameters characterizing sterically favored structures with high local packing capability, and then access structure-dynamics correlation by a novel nonlocal approach. We find that the particle mobility is under control of the static order parameter field. The fast $\beta$ process is controlled by the instantaneous order parameter field locally, resulting in short-time particle-scale dynamics. Then the mobility field progressively develops with time $t$, following the initial order parameter field from disorder to more ordered regions. As is well known, the heterogeneity in the mobility field (dynamic heterogeneity) is maximized with a characteristic length $\xi_{4}$, when $t$ reaches the relaxation time $\tau_{\alpha}$. We discover that this mobility pattern can be predicted solely by a spatial coarse graining of the initial order parameter field at $t=0$ over a length $\xi$ without any dynamical information. Furthermore, we find a relation $\xi \sim \xi_{4}$, indicating that the static length $\xi$ grows coherently with the dynamic one $\xi_{4}$ upon cooling. This further suggests an intrinsic link between $\tau_{\alpha}$ and $\xi$ : the growth of the static length $\xi$ is the origin of dynamical slowing-down. These we confirm for the first time in binary glass formers both in two and three spatial dimensions. Thus, a static structure has two intrinsic characteristic lengths, particle size and $\xi$, which control dynamics in local and nonlocal manners, resulting in the emergence of the two key relaxation modes, fast $\beta$ and slow $\alpha$ processes, respectively. Because the two processes share a common structural origin, we can even predict a dynamic propensity pattern at long timescale from the fast $\beta$ pattern. The presence of such intrinsic structure-dynamics correlation strongly indicates a thermodynamic nature of glass transition.

DOI: 10.1103/PhysRevX.8.011041

\section{INTRODUCTION}

Glasses are ubiquitous in nature and have a wide range of applications in our daily lives as well as in modern technology, thanks to the unique physical and chemical properties distinct from the crystal counterparts. Despite thousands of years of experience, however, the mechanism of the rapid increase in the viscosity and the emergence of complex dynamics of glass-forming liquids near the glass transition remains a major unsolved problem in condensed matter physics and materials science [1-8]. A major

\footnotetext{
*tanaka@iis.u-tokyo.ac.jp
}

Published by the American Physical Society under the terms of the Creative Commons Attribution 4.0 International license. Further distribution of this work must maintain attribution to the author(s) and the published article's title, journal citation, and DOI.
Subject Areas: Chemical Physics, Soft Matter, Statistical Physics mystery of the glass transition lies in the fact that, upon the transition, the emergence of the solidity is accompanied by little change in the geometric structure, as measured by the two-point correlation functions like the radial distribution function $g(r)$ or the static structure factor $S(q)$ (see, for example, Ref. [4]). This is in marked contrast to another type of liquid-solid phase transition, i.e., crystallization, where the solidity emerges as a result of the formation of ordered structures, i.e., long-range translational order [9]. A further mystery lies in the dynamic heterogeneity of a supercooled liquid observed in both experiments and simulations [10], whose length scale tends to diverge when approaching the glass transition. While this points to a similarity between the dynamic arrest of glass-forming liquids and the critical slowing-down in critical phenomena [11-15], there are crucial differences between them: the dynamic heterogeneity is detected through dynamic variables so the corresponding length scale is dynamical in its 
nature. Furthermore, unlike ordinary critical phenomena, the glassy dynamics slows down even at a microscopic (particle) level, which results in the emergence of the universal two-step relaxation.

The difficulty to uncover the mystery of glassy dynamics and glass transition is therefore linked to the following fundamental issues: (i) how to characterize the subtle growing structural order, which is sometimes named amorphous order or glassy order, if it exists, and (ii) how it is correlated to the complex dynamics, including two-step relaxation, dynamic heterogeneity, and drastic slowingdown [5]. Although the presence of a growing static length scale with direct correlation to dynamic heterogeneity was suggested for some glass formers $[13,14,16,17]$, its generality is a matter of debate. A serious conflict arises from results on binary glass formers indicating a distinct decoupling between them [18-25]. Furthermore, the characteristic relaxation time is related to the correlation length of the order parameter fluctuations via dynamic scaling for critical phenomena [9], but such a relationship between the static length and dynamics has not yet been established for glass transition. Resolution of these questions is expected to largely improve our understanding of the glassy phenomena and provide a proper theoretical description of the glass transition. Here it is worth noting that, although not direct, there is a rigorous inequality between the time scale $\tau_{\alpha}$ and the length scale $\xi$ [26]: $\tau_{\alpha} \leq \tau_{0} \exp \left(\right.$ const $\left.\times \xi^{d}\right)$, where $\tau_{0}$ is the microscopic time scale, $d$ is the dimensionality of the system, and $\xi$ is the longest correlation length in the system. This relation comes from the fact that the slowest possible relaxation mechanism would be a thermally activated process whose largest possible activation energy (for particles with finite-ranged interactions) would scale like $\xi^{d}[15]$.

Since the pioneering works by Widmer-Cooper and coworkers $[27,28]$, many pieces of evidence have accumulated for the correlation between static structure and dynamic heterogeneity [29-31]; however, the structural features behind it and the degree of the correlation have remained elusive until now. Logically, it is natural to expect higher mobility in more disordered structures. But simple structural quantities like the local free volume, the local potential energy, and the pair free energy have shown little correlation with the heterogeneous dynamics [16,28,32]. Large amounts of effort have been devoted to the identification of local structural order to compare with dynamics, such as icosahedral [33,34], crystal-like orders [13,14,16], or more complicated topological clusters (see Ref. [24] for a review). This has been an extremely difficult task due to the complex and temporally fluctuating nature of disordered liquid structures. While positive correlations between specific structures and glassy slow dynamics have indeed been observed in various simulation models and experimental systems, the relation between structures and dynamics has not been universal: Some studies reported almost one-to-one correspondence between structural order and dynamics $[13,14,16,17]$, whereas other studies reported a disparity between them [18-22] (see also Ref. [24] for a recent review). Even the qualitative correlation has recently been suggested to be highly system dependent [35]. Therefore, new approaches, e.g., going beyond a local correspondence between structure and dynamics, are necessary to clarify the mystery.

Instead of searching for particular structural orders, a number of approaches that are claimed as "order agnostic" have been proposed to detect the growth of amorphous order, e.g., point-to-set (PTS) correlations [18-20,36], finite-size scaling of entropy [21], vibrational-mode analysis of inherent states [22], and machine-learning analysis [31]. The common result is that the static length is distinct from and grows much slower than the dynamic one with decreasing temperature [18-23,25]. There are at least two possibilities to rationalize the situation: (1) additional mechanisms other than the structural ordering are important in the development of dynamic heterogeneity, and (2) other forms of structural order exist which control the glassy dynamics but remain undetectable by the above methods. Actually, the order-agnostic nature of PTS correlation lengths was recently questioned, for a polydisperse hard disk system, that the length scale associated with bondorientational order, rather than the PTS length, correlates with dynamics [17]. This result has triggered reconsideration of the pinning-protocol dependence in probing the PTS correlations [37], leading to debates about possible different natures of glass formers with and without mediumrange crystalline order [23,37]. To clarify the puzzle, further studies in the other classes of glass formers, e.g., binary systems widely used in previous studies [18-22], are necessary.

In this paper, we introduce a new nonlocal approach to tackle the classical problem of how the structure and heterogeneous dynamics are correlated in supercooled liquids. Three important types of glass formers with very different nature, namely, two-dimensional polydisperse and binary mixtures of soft disks and three-dimensional binary mixtures of soft spheres, are studied using molecular dynamics simulations. First, we introduce a set of new structural order parameters, which quantifies the capability to be efficiently packed locally. Then, using this order parameter, we establish an intimate structure-dynamics correlation for all three systems by revealing the underlying correspondence of time and length scales. In particular, the dynamic heterogeneity developed over the structural relaxation time $\tau_{\alpha}$ can be predicted with remarkable precision by a spatial coarse graining of the static order parameter field over a particular length scale $\xi$. Such strong structuredynamics correlations have so far never been reported for binary systems [18-22,24], mainly because the complex disordered structure has impeded the identification of the hidden structural ordering. Since the spatial coarse graining is purely a static operation, this result clearly indicates not 
only that the dynamic correlation length $\xi_{4}$ coincides with the static one $\xi$, but also that the glassy slow dynamics characterized by $\tau_{\alpha}$ is controlled by the static length scale $\xi$. We further show that the correlation of spatial heterogeneity in fast (short-time) and slow (long-time) dynamics is rooted in their common structural origin. These findings suggest a purely static origin for the dynamics of glassforming liquids including not only $\alpha$ but also fast $\beta$ relaxations.

The paper is organized as follows. In Sec. II, we introduce a set of new order parameters to detect the structural feature responsible for slow dynamics in both two and three dimensions. In Sec. III, we describe our simulation models and analysis methods for structure, dynamics, and their correlation. In Sec. IV, we first visualize the good correlation between structure and dynamics for both 2D polydisperse and binary systems (Fig. 2), which cannot be achieved in previous local approaches. This leads to the nonlocal scenario of structure-dynamics correlation in glass-forming liquids (Fig. 3). Key time and length scales are revealed through detailed analyses of the structure-dynamics correlation for the 2D and 3D systems, and possible theoretical descriptions of the data are discussed (Figs. 4 and 5). Structural contribution to dynamic fluctuations is disentangled to clarify the meaning of a causal correlation between structure and dynamics (Fig. 6). We further discuss the relation between fast and slow dynamics and demonstrate a common structural origin behind them (Fig. 7). Discussions about the role of order parameter and spatial coarse graining in characterizing the subtle structural order are given in Sec. V. Finally, we provide concluding remarks in Sec. VI.

\section{INTRODUCTION OF NEW STRUCTURAL ORDER PARAMETER OF GLASSINESS}

\section{A. Basic concept}

Here, we introduce a set of new structural order parameters in both $2 \mathrm{D}$ and $3 \mathrm{D}$, which can characterize many-body correlations responsible for slow dynamics. Our basic standpoint is that the structure of a supercooled liquid is controlled by the free energy. For a glass-forming system of hard spheres, for example, the free energy is determined by the steric constraint and the configurational entropy. For example, it is the steric constraint that is a driving force for hard-sphere crystallization. Thus, it is natural to expect that structural order should be associated with sterically favored structures. Provided that the density is basically homogeneous beyond the particle scale, such a sterically favored configuration should provide more room for particles, and hence, higher correlational (or, vibrational) entropy [14]. The validity of this last assumption has been confirmed by the absence of the correlation in the structure factor at the corresponding wave number. This physical picture should basically be valid for particle systems without directional bonding, which is justified by the absence of the growth of density fluctuations beyond the particle scale in such glass formers even with attractive interactions. Thus, we introduce a structural order parameter for such glass-forming systems, according to the strategy to seek a structural parameter that can characterize the capability to be efficiently packed locally.

Here it is worth stressing that the above-mentioned structural fluctuation has a quite asymmetric link to local dynamics: sterically favored regions have slow dynamics. Among a number of peculiar critical-like behaviors seen in glass-forming systems, this feature is unique to glassy criticality and absent in ordinary critical phenomena, in which the order parameter fluctuations do not have any coupling to local dynamics.

In this article, we consider three important model glass formers: weakly polydisperse and binary mixtures of soft disks in 2D and binary mixtures of soft spheres in 3D. The packing efficiency is determined by the configuration of a particle and its neighbors. In all three cases, the neighboring particles are defined according to the radical Voronoi tessellation [38].

\section{B. Order parameter for weakly polydisperse disks}

For weakly polydisperse disks, such a local structure with high packing capability can be described by the hexatic bond-orientational order parameter $\Psi_{6}$ [39], which has been proven to be effective in glassy systems with moderate strength of disorder in 2D [13,14,17]. This order parameter is linked to local sixfold rotational symmetry. For particle $j$ we have $\Psi_{6}^{j}=\left|\sum_{k} e^{6 i \theta_{j k}} / n_{j}\right|$, where $n_{j}$ is the number of nearest neighbors of particle $j$, and $\theta_{j k}$ is the angle of the bond $\mathbf{r}_{j k}=\mathbf{r}_{k}-\mathbf{r}_{j}$ with respect to the $x$ axis. $0 \leq \Psi_{6} \leq 1$, and ordered particles have larger $\Psi_{6}$.

\section{Order parameter for binary disk mixtures}

The local structure in binary mixtures of (soft) disks is more complex and obviously distinct from the crystalline order described by $\Psi_{6}$. In other words, the order parameter for binary mixtures is not linked to the breakdown of obvious spatial symmetry. Because of this difficulty, a wellestablished structural order parameter for such systems is still missing in the literature. On the basis of the above physical picture, here we introduce a new order parameter $\Theta$ for 2D binary disk systems. Considering a local configuration including a central particle $o$ and its neighbors, for each pair $\langle i j\rangle$ of neighboring particles next to each other, we measure the angle between $\mathbf{r}_{o i}$ and $\mathbf{r}_{o j}$, indicated as $\theta_{i j}^{1}$ in Fig. 1(a). Such triangles of three neighboring particles are considered as basic structural units in 2D. The reference configuration with these three particles perfectly just in touch is plotted in Fig. 1(b), with the central angle indicated as $\theta_{i j}^{2}$. Then we define the order parameter for particle $o$ as 

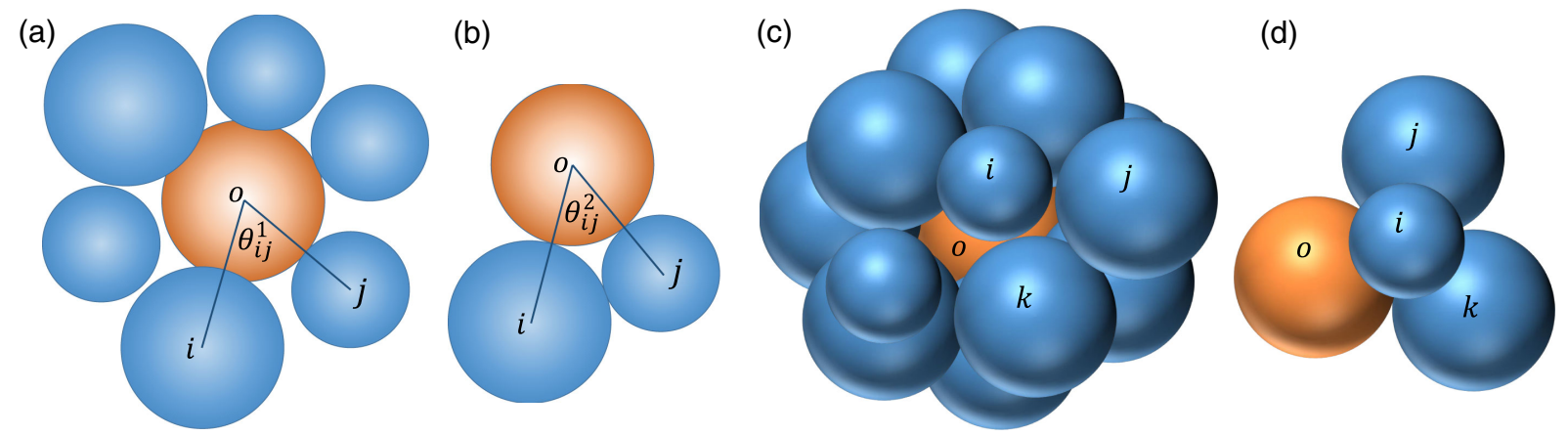

FIG. 1. Definition of the structural order parameter for binary systems. Left two panels: 2D binary disk system. (a) Illustration of a local configuration with a central particle $o$ surrounded by its six neighbors, two of which are indicated as $i$ and $j$. (b) Reference configuration with the three indicated particles just in touch. The definitions of central angles $\theta_{i j}^{1}$ and $\theta_{i j}^{2}$ are illustrated in (a) and (b), respectively. Right two panels: 3D binary sphere system. (c) Illustration of a local configuration with a central particle $o$ surrounded by its 14 neighbors, three of which are indicated as $i, j$, and $k$. (d) Reference configuration with the four indicated particles just in touch. See text for detailed definition of the structural order parameters.

$$
\Theta_{o}=\frac{1}{N_{o}} \sum_{\langle i j\rangle}\left|\theta_{i j}^{1}-\theta_{i j}^{2}\right|
$$

Here, $N_{o}$ is the number of pairs of neighbors which equals the number of neighbors of particle $o$, and the summation runs over all pairs of neighbors that are next to each other. $\Theta$ measures the deviation from sterically favored structures, i.e., the deviation of a local packing from the arrangement in which neighboring particles are most efficiently packed around the central particle. Larger values of $\Theta$ indicate stronger deviations from sterically favored configurations, i.e., less ability to be packed efficiently, and hence more disordered. We have confirmed that this order parameter can also be used for polydisperse disk systems, but here we employ the hexatic order parameter for polydisperse systems, which we already studied previously $[13,14]$, to show the power of this new method connecting structure and dynamics developed on the basis of the same physical concept.

\section{Order parameter for binary sphere mixtures}

Next, we construct the structural order parameter $\Omega$ for binary mixtures of (soft) spheres in 3D with the same spirit as in the $2 \mathrm{D}$ case, but the situation is much more complicated. In the case of systems with one type of spheres, there is a general approach to unambiguously partition the space into basic structure units of four particles, that is, a tetrahedron. This is done using the Voronoi-Delaunay tessellation, well known in both physics and mathematics [40]. The tetrahedron with vertices in these four spheres is called the Delaunay simplex. However, such a process is not straightforward in binary mixtures. The corresponding Delaunay tessellation does not exist dual to the radical Voronoi tessellation, and simple generalization gives miscounting. Thus, we develop the following procedures to characterize the local structural order in 3D. (1) The coordination shell is identified according to the radical Voronoi tessellation. An example of such local configuration is illustrated in Fig. 1(c), including the central particle $o$ and its neighbors. (2) Each three neighbors, which contribute to a vertex in the Voronoi cell of the central particle (the three Voronoi faces contributed by these three particles intersect at a Voronoi vertex), are identified together with the central particle to be a tetrahedron. This is key to correctly partition the local configuration into units of tetrahedra: not all of groups of three particles are necessarily selected, but only the ones which share a vertex according to the radical Voronoi tessellation. An example of such selection is indicated in Fig. 1(c). The lengths of each edge of this tetrahedron are denoted as $r_{o i}, r_{o j}$, etc. (3) The reference tetrahedron with these four particles perfectly just in touch is then constructed, as shown in Fig. 1(d). The edge lengths are denoted as $\sigma_{o i}, \sigma_{o j}$, etc. (4) The irregularity of a tetrahedron in the original configuration is measured as

$$
\omega_{\langle o i j k\rangle}=\frac{\sum_{\langle a b\rangle}\left|r_{a b}-\sigma_{a b}\right|}{\sum_{\langle a b\rangle} \sigma_{a b}} .
$$

Here, $\langle a b\rangle$ runs over the six edges of the tetrahedron $\langle o i j k\rangle$. (5) Finally, the structural order parameter of particle $o$ is calculated as

$$
\Omega_{o}=\frac{1}{N_{o}^{\text {tetra }}} \sum_{\langle o i j k\rangle} \omega_{\langle o i j k\rangle},
$$

where $N_{o}^{\text {tetra }}$ is the total number of tetrahedra surrounding particle $o$ and the summation is performed over all these tetrahedra. Similar to $\Theta$ in $2 \mathrm{D}, \Omega$ measures the deviation from sterically favored structures, or the deviation of a local packing from the perfectly packed arrangement around the central particle. Larger values of $\Omega$ indicate stronger deviations and more disordered. We stress that $\Omega$ (or $\omega_{\langle o i j k\rangle}$ ) is mainly contributed by the irregularity of bond-orientational order, rather than a simple extension or 
compression at fixed shape, because no obvious density fluctuation is observed in our systems.

\section{METHODS}

\section{A. Models and simulation methods}

We perform molecular dynamics simulations of three types of model glass formers: both polydisperse and binary mixtures of soft disks in 2D, and binary mixtures of soft spheres in 3D. The interaction between particle $i$ and $j$ is given by $V\left(r_{i j}\right)=\epsilon\left(1-r_{i j} / \sigma_{i j}\right)^{2} / 2$ for $r_{i j}<\sigma_{i j}$ and zero otherwise. Here, $r_{i j}$ is the separation between particles $i$ and $j$ and $\sigma_{i j}$ is the sum of their radii. For the polydisperse case, we introduce a Gaussian distribution of particle size with polydispersity $\Delta=\sqrt{\left\langle\sigma^{2}\right\rangle-\langle\sigma\rangle^{2}} /\langle\sigma\rangle$ and set the averaged diameter $\langle\sigma\rangle$ as the length unit. We choose $\Delta \geq$ $11 \%$ to avoid crystallization. We mainly present the results for $\Delta=11 \%$, but confirm basically the same results for $\Delta=12 \%$ and $13 \%$. For the binary case, large and small particles with equal number have a diameter ratio of 1.4 , with the small particle diameter $\sigma_{s}$ being the length unit. For all three cases, all particles have the same mass $m$. The energy, time, and temperature are in units of $\epsilon, \sqrt{m \sigma^{2} / \epsilon}(\sigma$ represents $\langle\sigma\rangle$ or $\sigma_{s}$ ) and $\epsilon / k_{B}$, with $k_{B}$ being the Boltzmann constant. We fix the volume fraction $\phi=$ $0.91\left(=\sum_{i=1}^{N} \pi \sigma_{i} / 4 L^{2}\right)$ in $2 \mathrm{D}$, and $\phi=0.6615915$ $\left(=\sum_{i=1}^{N} \pi \sigma_{i}^{3} / 6 L^{3}\right)$ in $3 \mathrm{D}$. This volume fraction in $3 \mathrm{D}$ corresponds to a number density of $\rho=0.6759715$ for which PTS correlations have been computed [19], and therefore facilitates a direct comparison of the PTS length scale with others. The number of particles is set to $N=10000$ for the polydisperse case in 2D and 4096 for the binary cases both in 2D and 3D. Simulations are performed in square boxes in 2D and cubic boxes in 3D, with periodic boundary conditions in the NVT ensemble.

\section{B. Spatial coarse graining of the order parameters}

We introduce systematic coarse graining to pick up the correlated nature of structural ordering. The coarse graining of order parameter $X$ (here, $X$ represents $\Psi_{6}, \Theta$, or $\Omega$ ) for particle $i$ is calculated by averaging over all particles within a length $L: \bar{X}_{i}(L)=\left[\sum_{j} X_{j} P\left(\left|\mathbf{r}_{j}-\mathbf{r}_{i}\right|\right) / \sum_{j} P\left(\left|\mathbf{r}_{j}-\mathbf{r}_{i}\right|\right)\right]$. We use an exponential core $P(x)=\exp (-x / L)$, under the hypothesis that the influence of the local structure decays exponentially in space. This provides a better consistency of the time and length scales (see Fig. 5), compared to other forms of coarse-graining cores like $P(x)=1$ and $P(x)=$ $\exp \left[-(x / L)^{2}\right]$. Discussions on the power of coarse graining are given in Sec. V B.

\section{Characterization of dynamics}

For 2D systems, there exist long-wavelength vibrational motions, known as Mermin-Wagner-type fluctuations [41-44], yet which are irrelevant to the structure relaxation if we properly characterize the dynamics. Therefore, we characterize the dynamics by using relative positions $\underline{\mathbf{r}}_{j}(t)=\mathbf{r}_{j}(t)-\sum_{k} \mathbf{r}_{k}(t) / n_{j}$, where the summation goes over all neighbors of particle $j$. Note that the neighboring particles are always defined at the initial configurations $t=0$. General features of glassy dynamics, e.g., the twostep relaxation, are recovered by such analyses in 2D, which are shown in Figs. S1 and S2 of the Supplemental Material [45]. We report results based on direct motions in Appendix B to show the importance of removing MerminWagner-type fluctuations in the analysis. For 3D systems, the dynamics are characterized using the original positions as usual. In the following, we use the notation of 2D relative positions in the formulas, which may be directly applied in 3D by using the direct positions.

The dynamic propensity $\langle\Delta r\rangle$ is defined in the isoconfigurational ensemble $[27,28,30]$. After the equilibration run, we quench the system to the nearest inherent structure by using the FIRE algorithm [46] to remove the random initial displacements. Starting from this initial configuration, 100 trajectories are simulated with different momenta assigned randomly from the appropriate Maxwell-Boltzmann distribution. The dynamic propensity of particle $i$ is then calculated through the isoconfigurational average: $\langle\Delta r\rangle_{i}(t)=\left\langle\left|\underline{\mathbf{r}}_{i}(t)-\underline{\mathbf{r}}_{i}(0)\right|\right\rangle_{\text {iso. }}$. Instead of particle motion in one realization, the dynamic propensity measures the probability of a particle to undergo a substantial displacement within a time interval. Twenty and thirty initial configurations are used in the calculations to ensure good statistics (see Figs. 4-6) for polydisperse and binary cases, respectively. We confirm good convergence by comparing the data with those from 200 trajectories and more initial configurations.

We disentangle the structural and purely dynamical contributions to the dynamic fluctuations within the isoconfigurational ensemble [29]. The dynamic fluctuations with a structural origin are calculated as $\Delta_{s}(t)=$ $\left\langle\sum_{i}\left\langle w_{i}(t)\right\rangle_{\text {iso }}^{2} / N\right\rangle_{\text {init }}-\left\langle\sum_{i}\left\langle w_{i}(t)\right\rangle_{\text {iso }} / N\right\rangle_{\text {init }}^{2}$, where $\langle\cdot\rangle_{\text {iso }}$ denotes the isoconfigurational average and $\langle\cdot\rangle_{\text {init }}$ is the average over all initial configurations. To monitor the structure relaxation, we use $w_{i}(t)=1$, if $\left|\underline{\mathbf{r}}_{i}(t)-\underline{\mathbf{r}}_{i}(0)\right|<b$, and zero otherwise, and set $b=0.15$. Accordingly, the overall fluctuation is given as $\Delta_{\text {tot }}(t)=\left\langle\sum_{i}\right.$ $\left.\left\langle w_{i}(t)^{2}\right\rangle_{\text {iso }} / N\right\rangle_{\text {init }}-\left\langle\sum_{i}\left\langle w_{i}(t)\right\rangle_{\text {iso }} / N\right\rangle_{\text {init }}^{2}$. The ratio $R_{f}(t)=$ $\Delta_{s}(t) / \Delta_{\text {tot }}(t)$ hence measures the relative amount of fluctuations with a structural origin.

\section{Rank correlation between structure and dynamics}

Our purpose is to measure the correspondence of structure and dynamic heterogeneity, i.e., whether particles in a more disordered environment tend to be more mobile. This is usually shown by pictures or quantified by the overlap of selected number of particles, which has a large degree of arbitrariness. Here we employ Spearman's rank correlation coefficient to quantify the correlation, which 
assesses the monotonic relationship (note its difference from a linear relation) between two variables without floppy parameters [47]. First the particles are sorted according to the structural order with the disordered ones in front and the ranks $O_{i}$ are assigned to each particle $i$. The same is done according to $\langle\Delta r\rangle$ with the mobile ones in front and the ranks $Q_{i}$ are assigned. The correlation is then calculated as $C_{r}=1-6 \sum_{i}\left(O_{i}-Q_{i}\right)^{2} / N\left(N^{2}-1\right)$. For the binary cases, we calculate the rank correlations for large and small particles separately and then average them. A monotonic increasing relationship between the two quantities gives $C_{r}=1$, whereas $C_{r}=0$ indicates the absence of the correlation.

\section{RESULTS}

\section{A. Nonlocal scenario for the correlation between structural order and dynamic heterogeneity}

First, we illustrate how key structural features responsible for dynamics emerge through progressive spatial coarse graining of an initial inherent structure and how it is correlated with the development of dynamic heterogeneity. For the ease of visualization, results are shown for $2 \mathrm{D}$ cases. The dynamics is investigated by calculating the averaged displacement amplitude $\langle\Delta r\rangle$, i.e., dynamic propensity, in the isoconfigurational ensemble (see Sec. III C). Time evolutions of $\langle\Delta r\rangle$ for polydisperse and binary cases are shown in Figs. 2(a) and 2(c), respectively. In both cases, particles with large dynamic propensity are very sparse at short times (see the first column), and then gradually form correlated clusters (see the second column). Thus, the mobility contrast becomes maximized (see the third column), then becomes obscure at very long times (see the fourth column), and finally should disappear. Note that the third column corresponds to $\langle\Delta r\rangle$ at around the $\alpha$ relaxation time $\tau_{\alpha}$. Therefore, the heterogeneous dynamics shows different spatial features at different timescales. This result indicates that, in addition to analyses focusing on a specific duration like the structure relaxation time $\tau_{\alpha}$, the full timescale is an important axis to unveil a complete relationship between structure and dynamics.

Corresponding to Figs. 2(a) and 2(c), respectively, the static structures at different coarse-graining lengths are shown in Figs. 2(b) and 2(d). In addition, the structural orders without coarse graining are given in Appendix A, Fig. 8. We find a strongly fluctuating nature at short length scales, correlated patterns at intermediate ones, and blurred patterns at long ones. The emergence of spatial correlation in the structure upon coarse graining, especially for the binary system, demonstrates the power of our approach in revealing the hidden structural ordering, which is masked by the superficially disordered structure, the thermal noise, and the currently limited knowledge of glassy order. This also suggests that the length scale is another crucial axis to consider the structure-dynamics correlation in supercooled liquids.
If we compare the bare (local) structural order parameter field with the dynamic propensity at $\tau_{\alpha}$, we can only see marginal correlation [e.g., for the binary case compare Fig. 8(b) with Fig. 2(c3)]. This clearly shows the inability of previous approaches searching for correlation between local structure and long-time $\alpha$ relaxation, which have hitherto left the structure-dynamics correlation undercover for the binary system and many others. However, strong structure-dynamics correlation is visible for the polydisperse (binary) case by comparing Fig. 2(a) [Fig. 2(c)] with Fig. 2(b) [Fig. 2(d)] in the same columns. In particular, we can see the best correspondence between Fig. 2(a3) [Fig. 2(c3)] and Fig. 2(b3)[Fig. 2(d3)] for the polydisperse (binary) case. This strongly indicates an intimate nonlocal link between static structural order and dynamic heterogeneity at the corresponding length and timescales.

We illustrate the nonlocal scenario of the structuredynamics correlation schematically in Fig. 3. Here we include the full time axis for dynamics with two important timescales, namely, the fast $\beta\left(\tau_{\beta}\right)$ and $\alpha$ relaxation times $\left(\tau_{\alpha}\right)$. In addition, the structural order is described in a full length axis, where two intrinsic length scales naturally emerge, i.e., the interparticle distance $(\sim \sigma)$ and static correlation length $\xi$. Different from previous local approaches (gray arrow), we propose that the structure-dynamics correlation exists in a nonlocal manner (vertical blue arrows): starting from an initial configuration, the dynamic field developed over time $t$ can be best predicted by structural information from a certain spatial range. In particular, the local structure controls the short-time $\beta$ relaxation, whereas the structural feature over its static correlation length $\xi$ controls the long-time $\alpha$ relaxation. This we show by Fig. 2 and demonstrate quantitatively in the following section. The important point is that the mobility field develops from an initial structure and systematically follows the initial order parameter field, spreading from the disordered to more ordered regions. This is different from the dynamic facilitation picture, where the most defective spots trigger initial movement and further motions facilitate randomly from these regions.

\section{B. Time and length scales in the structure-dynamics correlations}

Here, we quantify the correlation between structure and dynamics by looking at the tendency of more disordered particles being more mobile. In short, the correlations of the structural order at a coarse-graining length $L$ and $\langle\Delta r\rangle$ at time $t$ are calculated in order to pick up the relevant length scale in the static structure for the dynamic heterogeneity developed in a specific time range (see Sec. III D). Results are plotted in Figs. 4(a) and 4(d) for 2D polydisperse and binary systems, respectively, and Fig. 4(g) for the 3D binary system, in the complete spatial-temporal space 

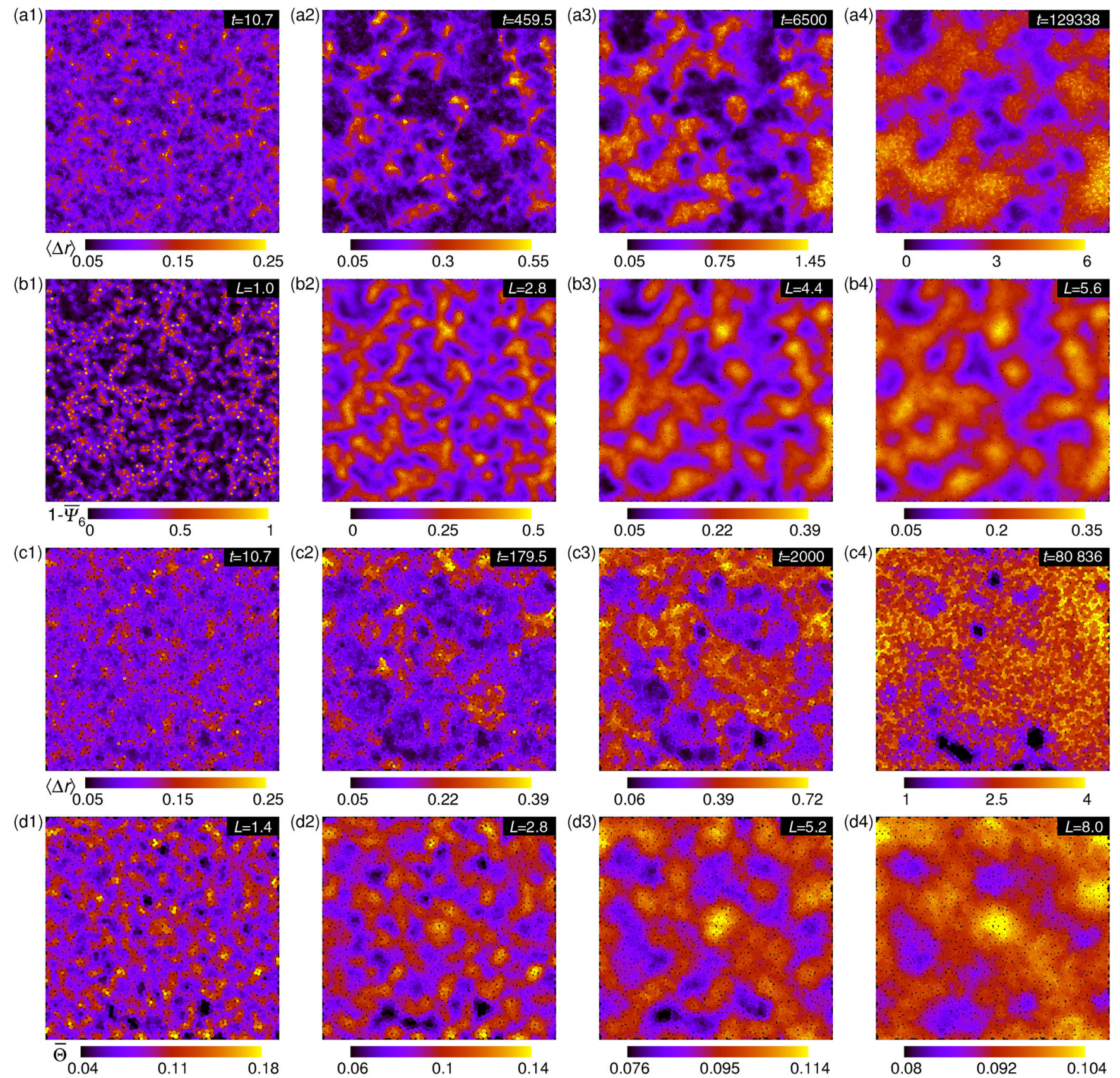

FIG. 2. Illustration of correlation between structural order and dynamic heterogeneity. Top two rows: 2D polydisperse disk system (polydispersity $\Delta=11 \%$ ) at $T=2 \times 10^{-3}$, with a corresponding $\alpha$ relaxation time $\tau_{\alpha} \approx 6541$. (a1)-(a4) Spatial distribution of dynamic propensity $\langle\Delta r\rangle$ at different timescales. (b1)-(b4) Spatial distribution of $1-\bar{\Psi}_{6}$ (the degree of disorder) of the initial configuration of the isoconfigurational ensemble for (a) at different coarse-graining length scales $L$. Bottom two rows: 2D binary disk system at $T=1.5 \times 10^{-3}$, with $\tau_{\alpha} \approx 1942$. (c1)-(c4) Spatial distribution of $\langle\Delta r\rangle$ at different timescales. (d1)-(d4) Spatial distribution of $\bar{\Theta}$ (the degree of disorder) for the same system as (c) at different coarse-graining length scales $L$. Note the clear resemblance in the same column, which indicates a structure-dynamics correlation at corresponding time and length scales. The similarity between the dynamic and static patterns is maximized for the third column for both polydisperse and binary systems (see text on its implication). See Secs. II and III for the definitions of structural order parameters and simulation details, and Appendix A, Fig. 8, for structural orders without coarse graining.

spanned by time $t$ and coarse-graining length $L$. The brighter color, which means better correlation, in the diagonal area confirms the nonlocal scenario suggested by Fig. 2 to be statistically valid.
Next, we extract more detailed information on the correlation. For the dynamic propensity at time $t$, we can identify a relevant length scale in the structure, i.e., the coarse-graining length $L_{p}$, which maximizes their 


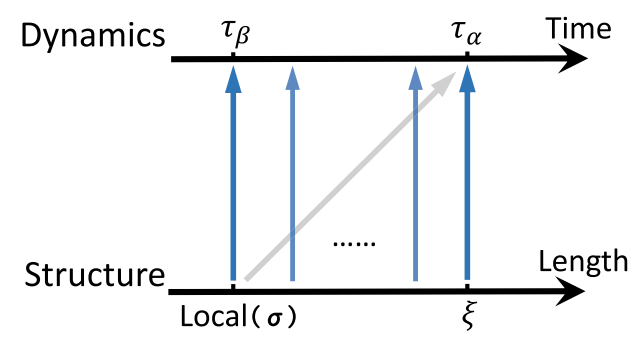

FIG. 3. Illustration of the nonlocal scenario of structuredynamics correlation. Top: The glassy dynamics evolves with time and shows two characteristic timescales, the fast $\beta\left(\tau_{\beta}\right)$ and $\alpha$ relaxation times $\left(\tau_{\alpha}\right)$. Bottom: The structure can be characterized by a static order parameter based on local configurations, which are intrinsically correlated over a characteristic length $\xi$. The nonlocal causal relation between structure and dynamics is represented by vertical arrows (blue), where the correspondences such as local structure $\sigma \rightarrow$ dynamics over $\tau_{\beta}$ and $\xi \rightarrow \tau_{\alpha}$ are highlighted. The previous approaches focusing on the correlation between local structure $(\sigma)$ and $\alpha$ relaxation are indicated by the inclined arrow (gray).

correlation. The values of the peak correlation $C_{r, p}$ and the corresponding $L_{p}$ are plotted as functions of time $t$ in Figs. 4(b) and 4(e) for 2D polydisperse and binary systems, respectively, and Fig. 4(h) for the 3D binary system. Interestingly, the initial strong correlation between structure and dynamics is established at fast $\beta$ timescale, as evidenced by the plateau or even a peak of $C_{r, p}(t)$ at $t \approx 10$. The corresponding coarse-graining length is at particle-size level. Further discussion on this fast $\beta$ process is given in Sec. IV D. While the correlation maintains at a high value until the $\alpha$ timescale, the relevant length scale of the structural order grows with time. This observation points to an intriguing relation between the structure and the development of dynamic heterogeneity, which definitely deserves further investigation in the near future. Moreover, we demonstrate the superiority of our nonlocal approach over the previous ones in Fig. 4(c), where the correlation of structural order and dynamic propensity $\langle\Delta r\rangle(t=4900)$ is plotted as a function of coarse-graining length $L$ [i.e., the vertical cut of Fig. 4(a) across the peak around $\tau_{\alpha}$ ]. $C_{r}$ is enhanced from 0.43 ( $L=0.5$ means bare structural order) to 0.76 at the peak after coarse graining. The same information for the 2D binary system (from 0.28 to 0.62) is conveyed by Fig. 4(f), and for the 3D binary system (from 0.35 to 0.54 ) by Fig. 4(i). From a theoretical view point, this big enhancement of structure-dynamics correlation also suggests that the $\alpha$ relaxation is intrinsically controlled in a nonlocal manner by the structure at the static correlation length $\xi$.

As shown in Fig. 4(a) and further illustrated by the black vertical arrow in Fig. 4(b) for the 2D polydisperse case, the correlation shows a peak that we identify at $t=\tau_{p, \Psi_{6}}$ and $L=\xi_{p, \Psi_{6}}$. Similarly, in Figs. 4(d) and 4(e) for the 2D binary case, we identify the peak at $t=\tau_{p, \Theta}, L=\xi_{p, \Theta}$, and in Figs. 4(g) and 4(h) for the 3D binary case at $t=\tau_{p, \Omega}$, $L=\xi_{p, \Omega}$. We have also independently calculated the structural relaxation time $\tau_{\alpha}$ through the intermediate scattering function, and the dynamic time $\tau_{4}$ and correlation length $\xi_{4}$ through the four-point density correlation functions (see Figs. S1-S6 in the Supplemental Material [45]). In Figs. 5(a) and 5(b) [Figs. 5(c) and 5(d)] for the 2D polydisperse [binary] case, and Figs. 5(e) and 5(f) for the $3 \mathrm{D}$ binary case, we plot the obtained time and length scales as a function of temperature $T$, respectively. The nice collapse of all data quantitatively confirms the intrinsic role of static structure in the glassy slow dynamics: (1) the dynamic heterogeneity develops following the underlying static structure, and hence, they share the same correlation length, and (2) there is a typical timescale for the dynamics to fully "feel" the influence of the structure, and that is why both $\tau_{4}$ and $\tau_{p, x}$ ( $x$ represents $\Psi_{6}$ and $\Theta$ for $2 \mathrm{D}$ polydisperse and binary systems, and $\Omega$ for the 3D binary system) follow $\tau_{\alpha}$, because $\tau_{\alpha}$ is the characteristic lifetime of local structures.

In Fig. 5(f), we also include the length scales computed in the same system using the PTS method (open symbols) by Kob et al. [19]. Apart from an overall coefficient, the temperature dependence of the PTS dynamic length $\xi_{s}^{\text {dyn }}$ (open circles) is consistent with our data within numerical precision; however, the PTS static length $\xi^{\text {stat }}$ (open diamonds) shows much milder temperature dependence and does not follow the growth of the dynamic one. It is based on this observation that the dynamic length scale was proposed to decouple with the static one and different natures were suggested between them [18-20]. This conflicts with our results, where the consistent growth of both static and dynamic length scales is observed. As in the 2D polydisperse hard disk system where the PTS static length is decoupled from the steeper growth of hexatic structural order and dynamic heterogeneity [17], our results seem to suggest that PTS also fails in detecting the subtle structural ordering in 3D binary mixtures, which is responsible for glassy slow dynamics. Since binary sphere systems are widely used as typical glass formers, our results strongly support a general structural origin of the dynamic heterogeneity and call for reconsideration of the order-agnostic nature of the PTS length. At even lower temperatures below the mode-coupling $T_{c}$, nonmonotonic temperature evolution of PTS dynamic length was observed [19]. It would be interesting to study the structure evolution using our order parameter in this temperature regime in the future.

For all three types of model glass formers, both 2D polydisperse and binary disk systems and the 3D binary sphere system, we find that the temperature dependence of timescales is well fitted by the Vogel-Fulcher-Tammann (VFT) function $\tau=\tau_{0} \exp \left[D T_{0} /\left(T-T_{0}\right)\right]$ [solid lines in Figs. 5(a), 5(c), and 5(e)]. Here, $T_{0}$ is the VFT glass transition (or Kauzmann) temperature and $D$ is the socalled fragility index. On the other hand, the temperature dependence of length scales can be fitted by the following 
(a)

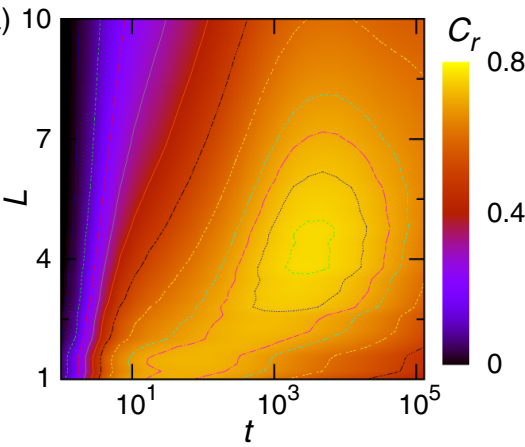

(d)

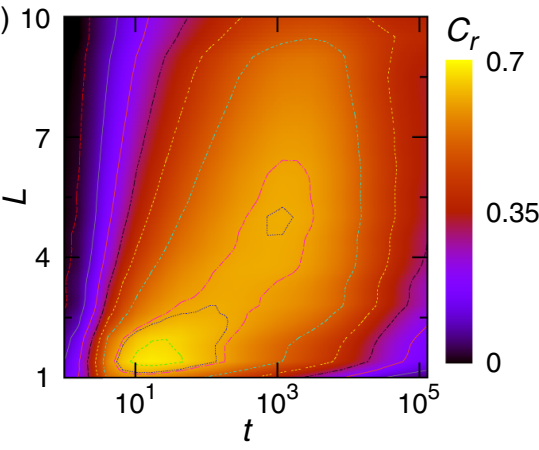

(g)

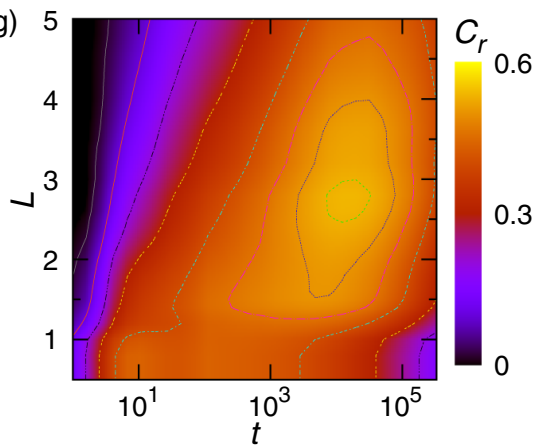

(b)

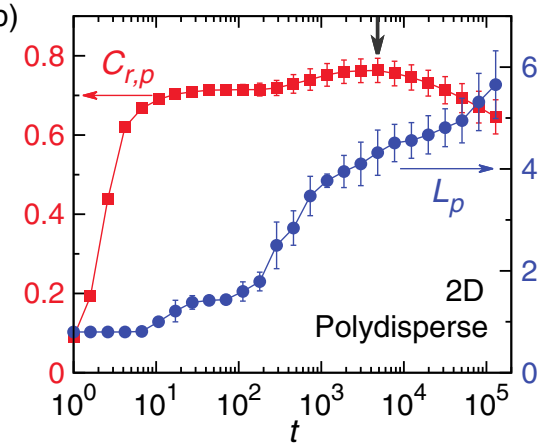

(e)

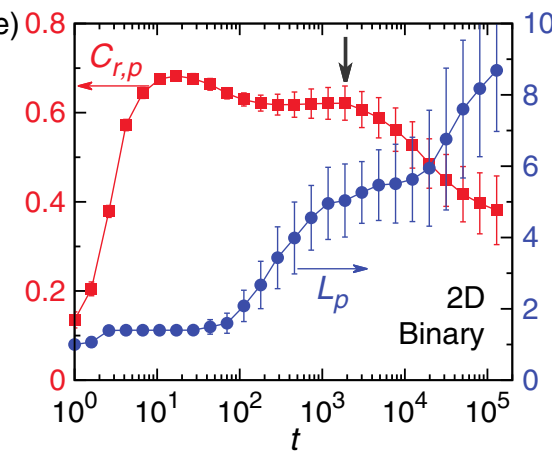

(h)

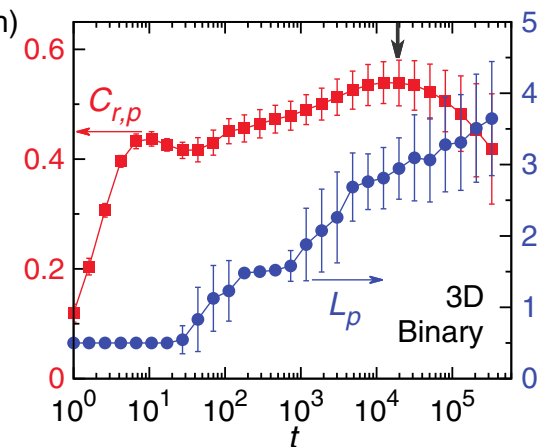

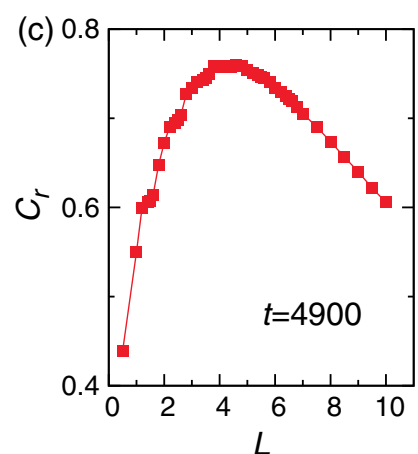

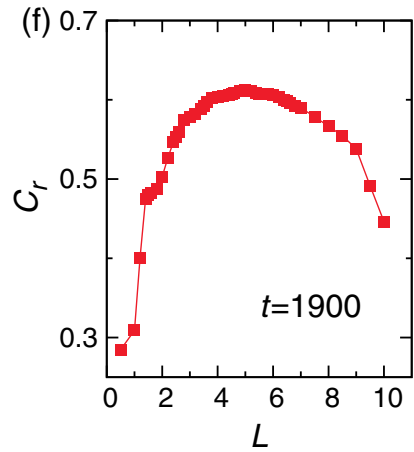

(i) 0

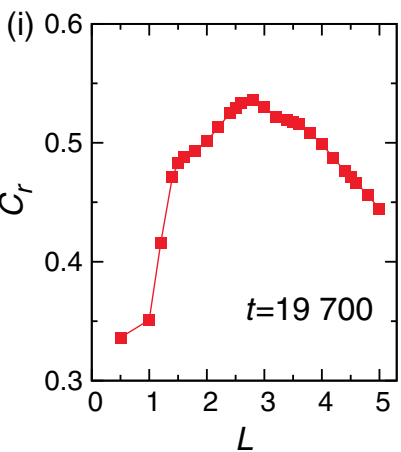

FIG. 4. Correlation between structural order and dynamic heterogeneity. Top: 2 D polydisperse system $(\Delta=11 \%)$ at $T=2 \times 10^{-3}$. (a) Cross-correlation $\left(C_{r}\right)$ of the structural order $\bar{\Psi}_{6}$ at different coarse-graining length $L$ with the dynamic propensity $\langle\Delta r\rangle$ at different time $t$. See Sec. III for the calculation of the cross-correlation. Note that the correlation shows a peak at $t=\tau_{p, \Psi_{6}}$ and $L=\xi_{p}, \Psi_{6}$. (b) The maximum structure-dynamics correlation $C_{r, p}$ and the corresponding coarse-graining length $L_{p}$ at each time $t$. The black vertical arrow indicates where the peak position, i.e., $\tau_{p, \Psi_{6}}$ and $\xi_{p, \Psi_{6}}$, is identified. (c) Change of $C_{r}$ as a function of coarse-graining length $L$ along the vertical cut of (a) across the peak around $\tau_{\alpha}$. The peak of $C_{r}$ highlights significant improvement compared to the local scenario without coarse graining. Middle: $2 \mathrm{D}$ binary system at $T=1.5 \times 10^{-3}$. (d) Cross-correlation between the structural order $\bar{\Theta}$ and $\langle\Delta r\rangle$. The peak of correlation at longer time and length scales is identified at $t=\tau_{p, \Theta}$ and $L=\xi_{p, \Theta}$. The appearance of peak correlation at short time and length scales in both (a) and (d) are discussed in Sec. IV D. (e),(f) The same as (b) and (c) for the 2D binary system. Bottom: 3D binary system at $T=6 \times 10^{-4}$. (g) Cross-correlation between the structural order $\bar{\Omega}$ and $\langle\Delta r\rangle$. The peak of correlation is identified at $t=\tau_{p, \Omega}$ and $L=\xi_{p, \Omega}$. (h),(i) The same as (b) and (c) for the 3D binary system. All data are from ensemble average, and error bars indicate the standard derivations.

power law [solid curves in Figs. 5(b), 5(d), and 5(f)]: $\xi=\xi_{0}\left[\left(T-T_{0}\right) / T_{0}\right]^{-2 / d}$, where $d$ is the spatial dimensionality $(d=2$ for weakly polydisperse and binary disk systems, whereas $d=3$ for binary sphere systems). Then we have made simultaneous fittings for the VFT relation of $\tau_{\alpha}$ and the power-law relation of $\xi$ and confirmed that a common ideal glass transition temperature $T_{0}$ gives reasonable fittings to both of them. The results of the fittings are quite satisfactory, although there are rather large standard deviations of the data. We further identify a scaling relation between the obtained length scale and the structural relaxation time: $\tau_{\alpha}=\tau_{0} \exp \left[D\left(\xi / \xi_{0}\right)^{d / 2}\right]$, which we show in the insets of Figs. 5(b), 5(d), and 5(f).

Here, we briefly mention the concept of activated dynamic scaling in disordered system [48], which rationalizes the above relationship between $\xi$ and $\tau_{\alpha}[14,15]$. In ordinary critical phenomena, the competition causing the critical point is between energy and entropy, and the 

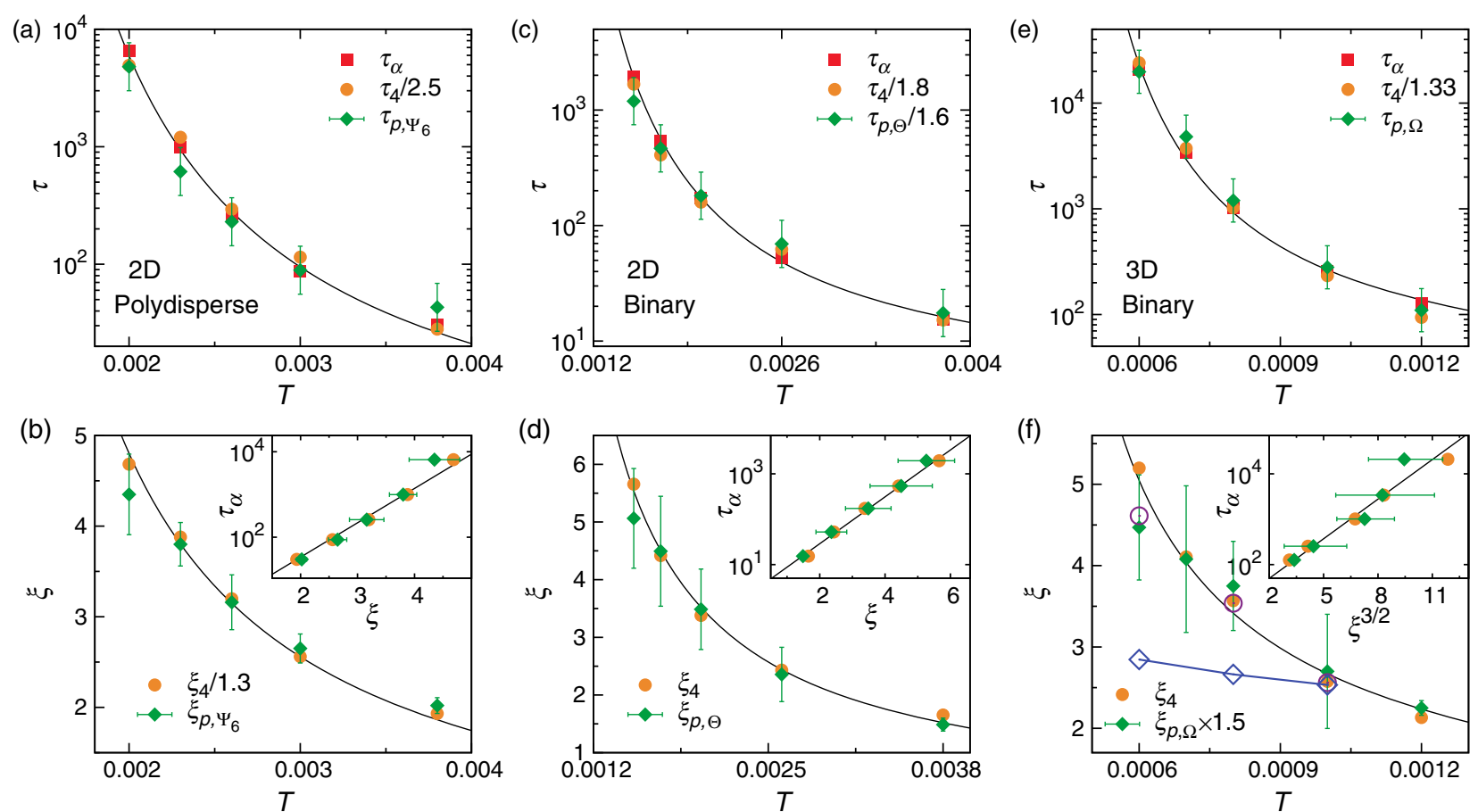

FIG. 5. Time and length scales in the structure-dynamics correlations. Left: $2 \mathrm{D}$ polydisperse system $(\Delta=11 \%)$. (a) Temperature dependences of the $\alpha$ relaxation time $\tau_{\alpha}$, the timescale of dynamic heterogeneity $\tau_{4}$, and $\tau_{p, \Psi_{6}}$. The solid line shows a VFT fit with $T_{0}=8.6 \times 10^{-4}$. (b) Temperature dependences of the dynamic length scale $\xi_{4}$ and $\xi_{p, \Psi_{6}}$. The solid line is a power-law fit with $\xi=\xi_{0}\left[\left(T-T_{0}\right) / T_{0}\right]^{-2 / d}$, where $d$ is the spatial dimension. Inset: Relationship of $\tau_{\alpha}$ to $\xi_{4}$ and $\xi_{p, \Psi_{6}}$. The solid line represents $\tau_{\alpha}=\tau_{0} \exp \left[D\left(\xi / \xi_{0}\right)^{d / 2}\right]$. (c),(d) The same analysis of temperature dependences of time and length scales as in (a) and (b) for the $2 \mathrm{D}$ binary system with $T_{0}=6.35 \times 10^{-4}$. (e),(f) The same analysis of temperature dependences of time and length scales as in (a) and (b) for the $3 \mathrm{D}$ binary system with $T_{0}=3.5 \times 10^{-4}$. In addition, we include the length scales from Ref. [19] by the PTS method in (f) for a comparison: the dynamic length $\xi_{s}^{\mathrm{dyn}} \times 1.75$ (open circles) and the static length $\xi^{\text {stat }} \times 4.6$ (open diamonds).

contribution of the latter to the free energy is always of the order of the thermal energy. Thus, the critical dynamics does not involve any large activation barriers. This leads to the power-law dependence of the relaxation time on the correlation length. In disorder systems, on the other hand, the dynamics in the correlation length $\xi$ is controlled by the internal frustration in energy itself: for example, competition between different steric preference in polydisperse systems. This leads to the activation energy scaled as $\xi^{\theta}$. This exponent $\theta$ is estimated as $\theta=d / 2$ from the following argument [15]: the characteristic correlation volume of length $\xi$ involves a number of particles proportional to $\xi^{d}$. Considering thermal fluctuations of individual particles, the correlation volume undergoes Gaussian fluctuations of a characteristic magnitude of $\xi^{d / 2}$, which sets the activation barrier.

The power-law relation of $\xi$ with $\left(T-T_{0}\right)$ and the relation between $\xi$ and $\tau_{\alpha}$ are consistent with two known scenarios: the Ising-type critical scenario for the glass transition [13-15] and the random first-order transition theory $[5,8,49]$. The key difference between the two scenarios is the relation between static and dynamic correlation length, $\xi$ and $\xi_{4}$ : The former predicts $\xi \cong \xi_{4}$. On the other hand, the latter predicts that the dynamical correlation length $\xi_{4}$ and the mosaic length $\xi$ diverge towards the mode coupling $T_{c}$ and $T_{0}$, respectively [19]. The divergence of $\xi_{4}$ is a consequence of a spinodal singularity at $T_{c}$, above which metastability is lost and, therefore, the mosaic picture is no longer valid $[5,49]$. Since $T_{c}>T_{0}, \xi_{4}$ should increase much more rapidly than $\xi$, which is not consistent with our observation. Thus, our observation is more consistent with the critical scenario, but further investigation is necessary for drawing a definite conclusion including a possibility of other scenarios. Nevertheless, the common behaviors of polydisperse and binary disk systems in 2D and binary sphere system in 3D, three important model glass formers with very different nature, strongly suggest the generality of an intimate correlation between structural ordering and glassy dynamics. We therefore speculate that all glass formers can be unified within this picture. The relevant structural ordering in glass transition may be sterically favored structures, no matter whether it is linked to crystalline order or subtle amorphous order, which does not show obvious symmetry breaking.

\section{Causal relation between structure and dynamics}

Here, it is worth noting that a causal link between structural and dynamic heterogeneity does not mean a 

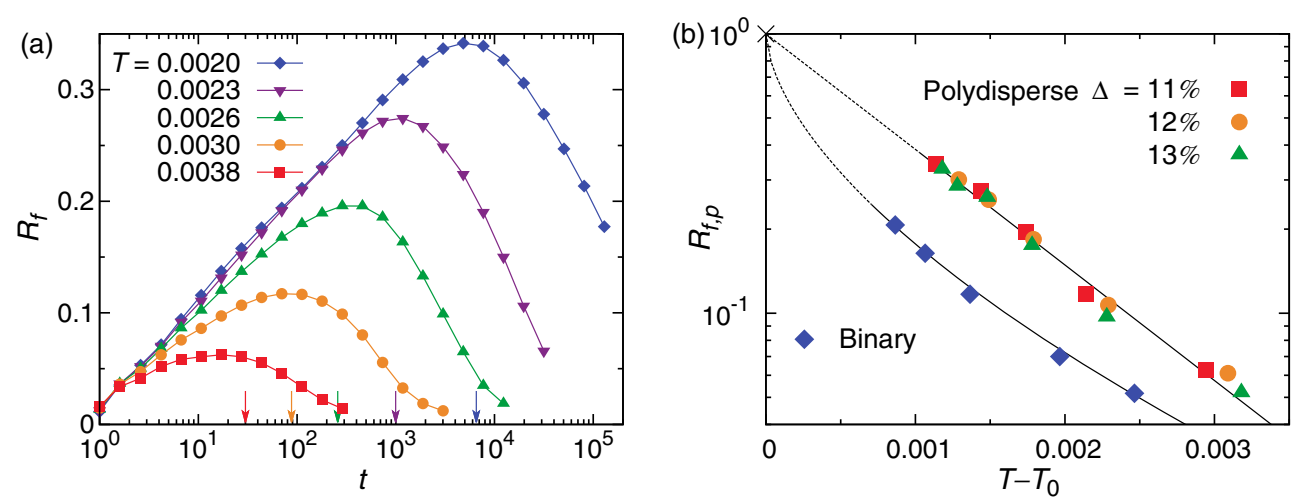

FIG. 6. Dynamic fluctuations with a structural origin. (a) The relative contribution from the structure to the overall dynamic fluctuations $R_{f}$ for the $2 \mathrm{D}$ polydisperse system $(\Delta=11 \%)$ at different $T$. $R_{f}$ is defined as the ratio of fluctuations with the structural origin to the overall value. See Sec. III for the detailed definition. The $\alpha$ relaxation time at each $T$ is indicated by the arrow with the same color. (b) Dependence of the peak value of $R_{f}, R_{f, p}$, on $T-T_{0}$. For 2D polydisperse systems, the data for the three systems with different polydispersities $\left(\Delta=11 \%, 12 \%\right.$, and 13\%) collapse on a common curve of $R_{f, p}(T)=\exp \left[-\left(T-T_{0}\right) / 0.00105\right]$. For the $2 \mathrm{D}$ binary system, on the other hand, the data are fitted by $R_{f, p}(T)=\exp \left\{-\left[\left(T-T_{0}\right) / 0.0004\right]^{0.6}\right\}$.

one-to-one determinant relation. This point has been raised in a number of studies [27-29] and is evident by comparing the real-space plot of the dynamic propensity and the displacements from one trajectory [27]. Actually, the static ordering determines the probability of a particle to be mobile or not, and the displacement in one run is controlled by structure in a statistical sense where the thermal fluctuations also play an important role. The consistence of the structural and dynamical correlation lengths shown in Fig. 5 indicates that the long-time and large-scale correlated motions are mainly determined by the structure (note that the $\xi_{4}$ is probed at $\tau_{4} \sim \tau_{\alpha}$ ). The fluctuations of purely dynamic origin are expected to be random and short ranged in both space and time. Still, it is interesting to quantify the relative amount of fluctuations with a structural origin over the total value $R_{f}(t)=\Delta_{s}(t) / \Delta_{\text {tot }}(t)$ [29]. Here we show results in two dimensions. From Fig. 6(a), we observe two major features: (1) $R_{f}$ shows a peak at around $\tau_{\alpha}$ and (2) the peak value of $R_{f}, R_{f, p}$, increases significantly with decreasing temperature and reaches $\sim 35 \%$ for the lowest $T$ in the study. The appearance of the peak further highlights the importance of the structural relaxation time $\tau_{\alpha}$ in the correlation between structure and dynamics. We find that for polydisperse systems $R_{f, p}$ grows with a decrease in temperature as $\ln R_{f, p} \propto-(T-$ $\left.T_{0}\right) \propto-\xi^{-1}$ commonly for three different polydispersities [see Fig. 6(b)]. Qualitatively, this relation may be reasonable since the relative importance of the order in the dynamics to the stochastic effect may be scaled by $\xi$. For the binary system, we find a different relation, which may stem from the presence of two types of particles with different mobilities. This point needs further study. It would be interesting to check whether $R_{f, p}$ really grows towards unity when approaching the ideal glass transition temperature in future works and reveal the physics behind the above empirical relation. We also make a similar analysis focusing on the fast $\beta$ process, and find a similar significant influence from a structure (see Appendix $\mathrm{C}$ for details).

\section{Common structural origin of fast and slow dynamics}

Next, we study the connection between the short-time (fast $\beta$ ) and long-time $(\alpha)$ dynamics. The fast cage-rattling dynamics was considered as a direct probe of initial configuration and its resemblance with slow $\alpha$ relaxation was taken as the evidence of structure-dynamics correlation [28]. On the contrary, it was also proposed that the fast dynamics is localized and the slow dynamics develops as a result of dynamic facilitation, which is purely of dynamic origin [50]. We construct unique simulation schemes to resolve this controversy. First, we pick up the dynamic propensity at short times, i.e., Fig. 2(a1), and spatially coarse grain it at different length scales. The marked resemblance between Figs. 7(a)-7(c) and Figs. 2(b2)-2(b4) proves that (the propensity of) fast dynamics contains similar structural information as the static order parameter; in other words, the fast $\beta$ dynamics is also controlled by the static order. In fact, the initial correlation of structural and dynamic heterogeneity builds up at the fast $\beta$ timescale [see Figs. 4(b) and 4(e) for the first peak or plateau of the cross-correlation]. Therefore, although being localized and highly fluctuating, the fast dynamics is actually cooperative in nature, in line with a recent work by Karmakar et al. [51]. Second, we stop the dynamics of the isoconfigurational runs at short times $t=10.7$ [corresponding to Fig. 2(a1)] and restart it by assigning a new velocity field from the Maxwell-Boltzmann distribution at 
(a)
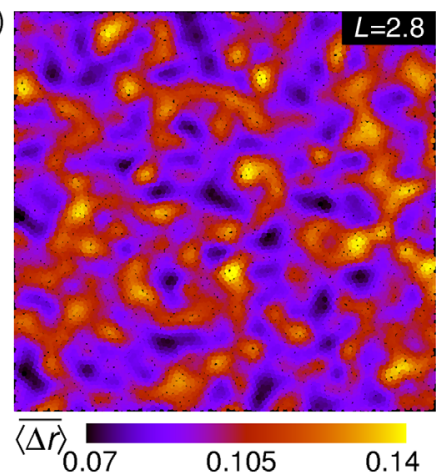

(d)

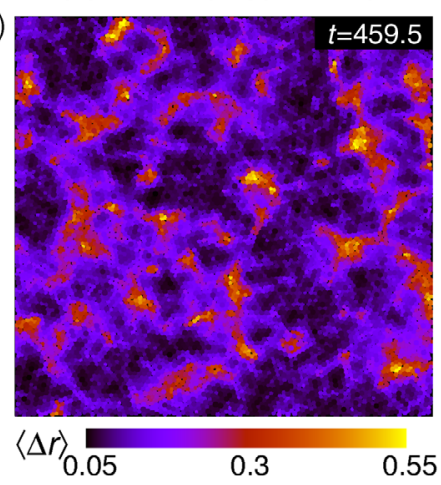

(b)
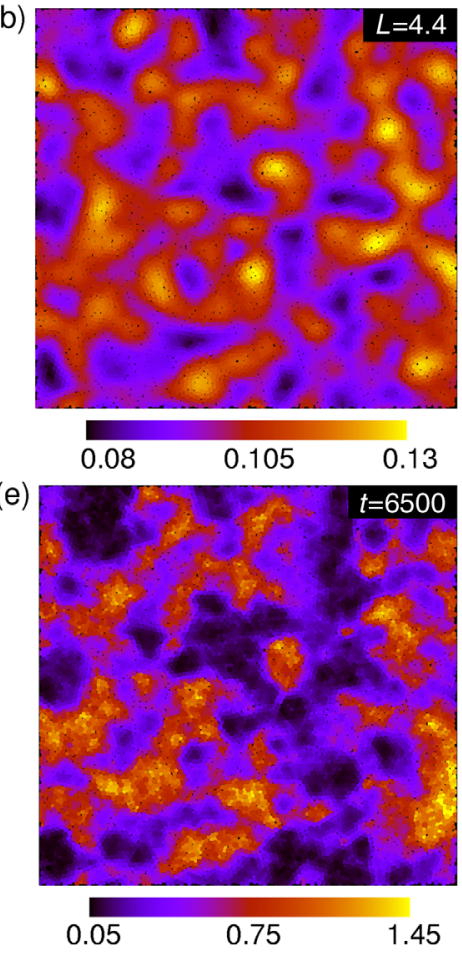

(c)
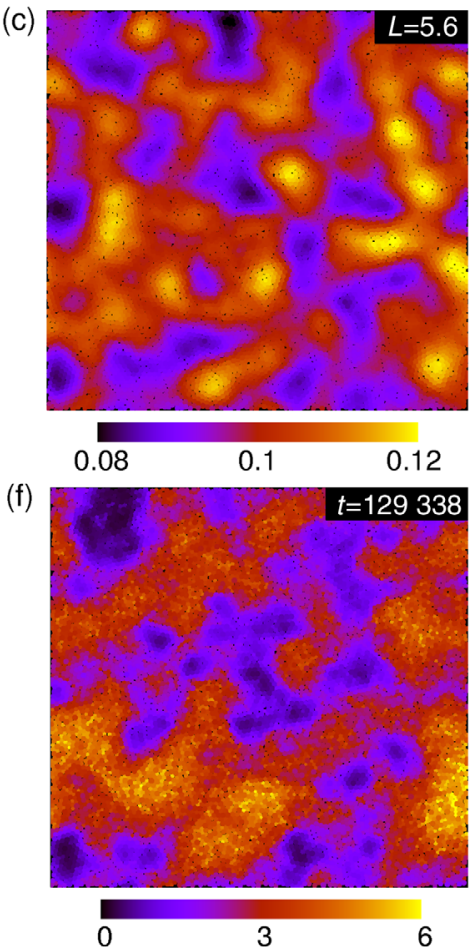

FIG. 7. Correlation of fast and slow dynamics. (a)-(c) Spatial distribution of dynamic propensity coarse grained from the one at $\beta$ timescale $t=10.7$ for the 2D polydisperse system $(\Delta=11 \%)$ at $T=2 \times 10^{-3}$ [i.e., Fig. 2(a1) for fast dynamics]. (d)-(f) Spatial distribution of dynamic propensity corresponding to Figs. 2(a2)-2(a4), but here the dynamics is stopped at $t=10.7$ and then restarted by assigning each particle a new velocity from the Maxwell-Boltzmann distribution at the same temperature. The new velocity field is different from and independent of the initial one, so the dynamic correlation before and after $t=10.7$ is totally eliminated after this operation. Note the clear resemblance between (a)-(c) [(d)-(f)] and Figs. 2(b2)-2(b4) [Figs. 2(a2)-2(a4)]. This indicates the common structural origin behind fast $\beta$ and slow $\alpha$ dynamics.

the same temperature. In this stop-and-go process, the connection between the short-time and long-time dynamics due to dynamical correlation is eliminated and the only remaining connection is from the structure. The recalculated dynamic propensities are shown in Figs. 7(d)-7(f). The obvious resemblance compared to Figs. 2(a2)-2(a4) strongly suggests that the close correlation between the fast and slow dynamics emerges as a natural result of the underlying structural order in common.

\section{DISCUSSIONS}

\section{A. Role of structural order parameters}

Here, we briefly discuss the physical meaning of structural order parameters (see also Sec. II). The first choice for the description of a liquid state is the density field $\rho(\mathbf{r})$ and its two-body correlation, which is the basic input of standard liquid-state theories including the modecoupling theory $[52,53]$. However, for supercooled liquids at high density, many-body correlation due to local structural ordering plays an important role which is intrinsically not captured by $\rho(\mathbf{r})$ [14]. Bond-orientational order parameters represent many-body correlations in a natural manner.
However, our newly defined order parameters for the binary mixture in both $2 \mathrm{D}$ and $3 \mathrm{D}$ have nothing to do with crystal-like bond-orientational order. We emphasize that what is important is that in any system a supercooled liquid tends to locally attain sterically favored structures, or structures of high packing capability. For monodisperse or weakly polydisperse systems, this sterically favored local structure is captured by crystal-like bond-orientational order (hexatic order described by $\Psi_{6}$ in our case). This is a consequence of natural symmetry selection under dense packing for nearly equal-size particles. For binary mixtures, on the other hand, this is no longer the case, and sterically favored structures are captured by our new structural order parameters $(\Theta$ in $2 \mathrm{D}$ and $\Omega$ in $3 \mathrm{D})$, which are not linked to any crystal-like symmetry but to more exotic amorphous order. The common feature of all three order parameters is that they can detect sterically favored local structures.

\section{B. Physical consequence of coarse graining}

In Sec. IV, we successfully unveil an intimate correlation between the structural and dynamic heterogeneity, despite the fact that we can neither construct a perfect order 
parameter to characterize the disordered structures, especially for the binary systems, nor know a perfect way to do the spatial coarse graining. The mechanism behind the robustness of our method can be explained as follows: A structural order parameter $X$, which is imperfect but somehow effectively picks up the relevant many-body correlations through orientational order, can be decomposed into two parts as $X=f\left(X_{p}\right)+X_{r}$. Here, $X_{p}$ is the unreachable perfect order parameter which is spatially correlated at length $\xi_{p} \gg 1 . f(\cdot)$ is some unknown function and $X_{r}$ is a trivial component which is irrelevant to glassy dynamics and supposed to be highly fluctuated with a correlation length $\xi_{r} \lesssim 1$. The construction of the order parameter $X$ has two consequences: (1) it is not necessarily feasible to extract the static correlation length directly from the spatial correlation function of $X$ and (2) upon coarse graining at a length scale $\xi>\xi_{r}$, the trivial component will be naturally blurred into a smooth background and the correlated feature from the nontrivial part will emerge and maximize at $\xi_{p}$.

Based on the above argument, the quality of an order parameter depends on the relative amount of its nontrivial component. We stress that an order parameter which appropriately captures the important many-body correlation is fundamental to reveal the structure-dynamics correlation, while our coarse-graining approach works to uncover this correlation which may be blurred by the imperfectness of the order parameter. In other words, the static length scale revealed here is intrinsically embedded in the order parameter, rather than in the coarse-graining process. We illustrate this point in Appendix D by showing the failure of local potential energy $E$ as a structural order parameter.

Finally, we mention that time averaging was previously employed to remove thermal noise and unveil structural order (see, e.g., Ref. [13]). This is an effective way to remove thermal noise, but the structural order extracted in this way can no longer be regarded as a purely static order in a strict sense and the amount of dynamic information thus included is without control. In a practical sense, it would be interesting to clarify the effect of time averaging systematically, by using our spatial coarse graining as a reference.

\section{CONCLUDING REMARKS AND SUMMARY}

To summarize, herein we propose a new nonlocal scenario to study the role of structural order in glassy dynamics and successfully unveil an intimate correlation between the structural and dynamic heterogeneity for both fast and slow dynamics. This we show by visualizing that the dynamic propensity field develops from most disordered regions of the initial structure systematically following the coarse graining of the order parameter field, and quantitatively by confirming that the dynamical correlation length coincides with the static correlation length which maximizes the structure-dynamics correlation around $\tau_{\alpha}$. The results are consistent for three types of glass formers, polydisperse and binary disk systems in 2D and a binary sphere system in 3D, implying the generality of the conclusion. We emphasize that, for binary glassforming liquids, especially in 3D, such a direct and nearly one-to-one structure-dynamic correlation has remained undercover despite decades of efforts $[24,27,28,35]$. Since the growth of structural order is of thermodynamic origin, our finding suggests that the glassy slow dynamics is influenced by the thermodynamics, pointing to a thermodynamic nature of glass transition (see also Sec. II). Our new structural order parameter and nonlocal approach can be easily implemented in colloidal experiments with single-particle resolutions, and therefore we hope that our work may trigger further experimental studies to reveal the nonlocal structure-dynamics correlation in real systems [44,54-57].

The bond-orientational order parameter and our new order parameters we employ in this study commonly characterize sterically favored structures. This suggests that the structural order parameter of glass transition may be generally linked to sterically favored structures for systems without directional bonding. This looks physically natural on noting that vitrification is induced by high packing. Since packing is intrinsically a consequence of many-body effects, our study indicates the essential role of many-body correlations in glass transition.

The power of our method is rooted in the fact that some subtle structural order indeed develops when glass-forming liquids are supercooled approaching the glass transition. If the cooperative nature of glassy dynamics is a consequence of the underlying structural order, then a natural step is not only to look at the local structure but also to pick up the structural order at a longer length scale, e.g., the static correlation length. As evidenced by our results (Fig. 2), hierarchical structural features emerge upon spatial coarse graining which are hardly visible at a local level. Our spatial coarse-graining procedure reveals the presence of the two key length scales, which govern the dynamics of glass-forming liquids: a structural feature at a short length scale controls the short-time $\beta$ relaxation, whereas that at a mesoscopic length scale $\xi$ controls the long-time $\alpha$ relaxation. The important point is that both fast and slow dynamics are controlled by the same static order. This provides us with a new understanding of the universal twostep relaxation in supercooled liquids: The presence of the two timescales is a natural consequence of the presence of the two key spatial scales in supercooled liquids, the particle size $\sigma$ and the static correlation length $\xi$. The former is the unique fundamental scale of any "equilibrium" liquids and the latter is specific to a supercooled state. 
Finally, it is worthwhile to mention a fundamental remaining open question: what physical mechanism connects the diverging length scales with the glassy slow dynamics, or more specifically, how does the correlation between structural ordering and complex dynamics builds up? Our work can provide a strong hint for this: the characteristic relaxation time $\tau_{\alpha}$ is actually the time required for the progressive development of the mobility field characterized by the correlation length of the dynamic heterogeneity to reach the length $\xi: \ln \tau_{\alpha} \propto \xi^{d / 2}$ for the two $d=2$ and one $d=3$ systems studied. This implies that the structural relaxation $\tau_{\alpha}$ is determined by the spatial extent $\xi$ of the kinetically activated cooperative processes. We hope that our finding will initiate further studies on this fundamental problem.

\section{ACKNOWLEDGMENTS}

We thank John Russo for a critical reading of our manuscript. This study was partly supported by Grantsin-Aid for Specially Promoted Research (Grant No. 25000002) from the Japan Society for the Promotion of Science (JSPS) and a JSPS Postdoctoral Fellowship for H. Tong.

\section{APPENDIX A: SPATIAL DISTRIBUTION OF ORDER PARAMETERS WITHOUT COARSE GRAINING}

Figure 8 shows the spatial distribution of order parameters $\Psi_{6}$ and $\Theta$ without coarse graining for $2 \mathrm{D}$ polydisperse and binary systems, respectively. The highly fluctuated nature, especially for $\Theta$ in the binary system, is obvious compared to Figs. 2(b) and 2(d) after coarse graining. Therefore, the correlated nature of the structure may be masked due to the imperfectness of the order parameter at the local level.
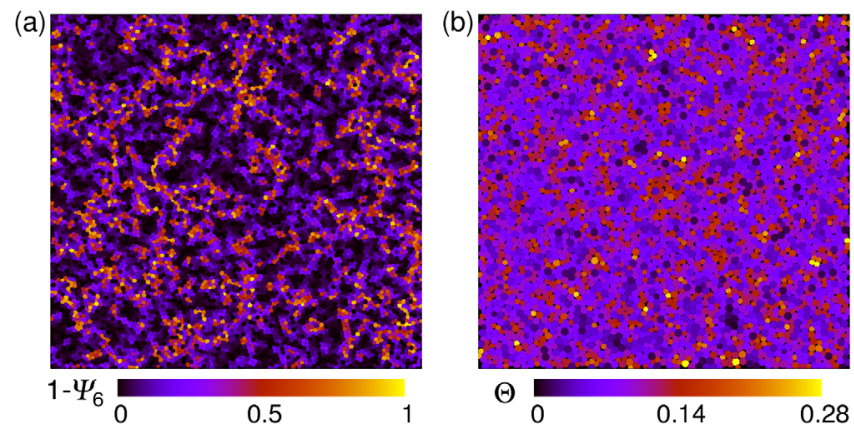

FIG. 8. Spatial distribution of order parameters without coarse graining. (a) Spatial distribution of hexatic order $\Psi_{6}$ for an inherent state of 2D polydisperse systems at $T=2 \times 10^{-3}$, corresponding to Fig. 2(b). (b) Spatial distribution of order parameter $\Theta$ for an inherent state of 2D binary systems at $T=1.5 \times 10^{-3}$, corresponding to Fig. 2(d).

\section{APPENDIX B: TIME AND LENGTH SCALES IN THE STRUCTURE-DYNAMICS CORRELATIONS IN 2D ACCORDING TO DIRECT MOTIONS}

For 2D systems, there exist the Mermin-Wagner-type long-wavelength vibrations [41-44], which may mask the motions relevant to the structure relaxation. In the main text, we characterize the dynamics by using relative positions in 2D and successfully unveil the structural origin of dynamic heterogeneity. Here, we show results of time and length scales in the structure-dynamics correlations according to direct motions to quantity the influence of long-wavelength vibrations. In the calculation of four-point correlation functions of direct motions [see Eqs. (3) and (4) of the Supplemental Material [45]], the cutoff in the overlap function $Q(t)$ is set as $b=0.3$. Corresponding to Figs. 5(a) and 5(b), Fig. 9 shows the temperature dependences of time and length scales in the 2D polydisperse system according to direct motions. Obvious deviations are observed, especially for the length scales. This can actually be expected as following. Since the reversible long-wavelength vibrations are counted which overestimate the relaxation rate, we obtain a smaller structure relaxation time $\tau_{\alpha}$ and dynamic timescale $\tau_{4}$. In the meantime, while we expect faster relaxation of more defective particles, the part of motions from the long-wavelength vibrations are collective over much larger scale than the particle size. The inclusion of long-wavelength vibrations therefore has two consequences: (1) it masks the correspondence between structural order and relaxation dynamics, and influences the accuracy in the estimations of $\tau_{p, \Psi_{6}}$ and $\xi_{p, \psi_{6}}$, and (2) the long-range correlation of vibrational motion results in an overestimation of the dynamic length scale $\xi_{4}$. These lead to the discrepancy of both time and length scales, as shown in Figs. 9(a) and 9(b) respectively.

\section{APPENDIX C: SIGNATURES OF STRUCTURAL INFLUENCE ON FAST $\beta$ DYNAMICS}

In Sec. IV C, we show the relative contribution from the structure to the overall dynamic fluctuations $R_{f}(t)=\Delta_{s}(t) / \Delta_{\text {tot }}(t)$. As specified in Sec. III C, there we set the cutoff $b=0.15$ to monitor the $(\alpha)$ structure relaxation. Here, we choose smaller values of $b$ to magnify the signal of structural influence on the fast $\beta$ process, and the results for a $2 \mathrm{D}$ polydisperse system are shown in Fig. 10(a). Besides the main peak at around $\tau_{\alpha}$, a shoulder at short time $t \approx 10$ emerges with decreasing $b$, strongly indicating a significant influence from the structure at $\beta$ timescale.

To further illustrate the fast $\beta$ process, we plot the overlap function $q(t)$ in Fig. 10(b), which is defined as $q(t)=$ $\sum_{i} w_{i}(t) / N$ [see Sec. III C for the definition of $w_{i}(t)$ ]. The appearance of the two-step relaxation process is obvious. 

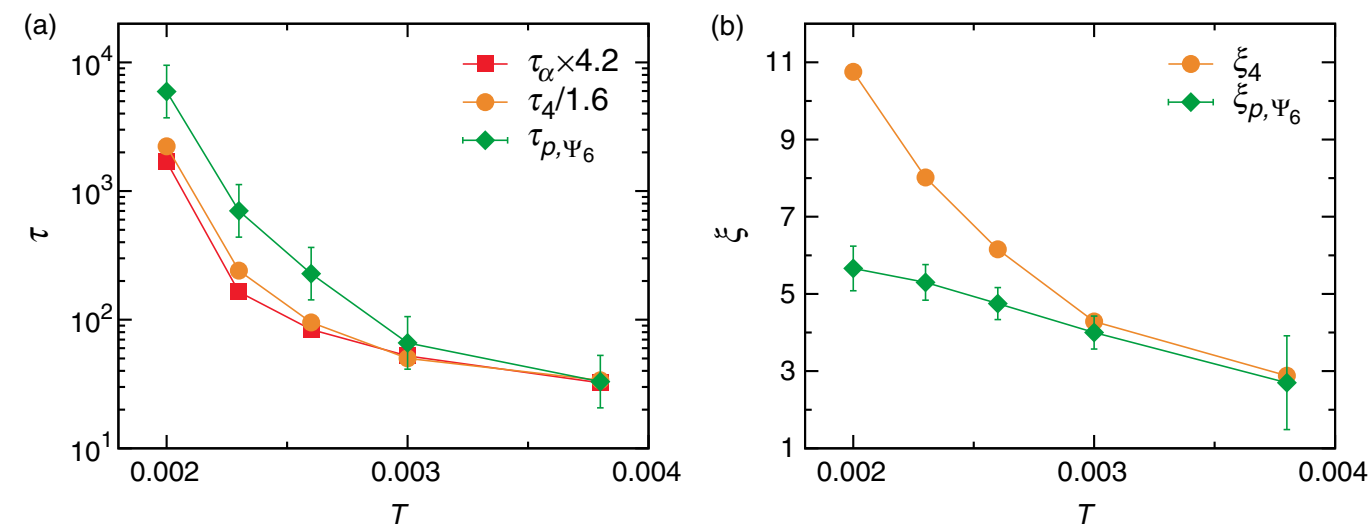

FIG. 9. Time and length scales in the structure-dynamics correlations according to direct motions in 2D polydisperse system ( $\Delta=11 \%$ ). (a) Temperature dependences of the $\alpha$ relaxation time $\tau_{\alpha}$, the timescale of dynamic heterogeneity $\tau_{4}$, and $\tau_{p, \Psi_{6}}$. (b) Temperature dependences of the dynamic length scale $\xi_{4}$ and $\xi_{p, \Psi_{6}}$. Note that here all quantities are from direct motions without removing the Mermin-Wagner-type fluctuations.
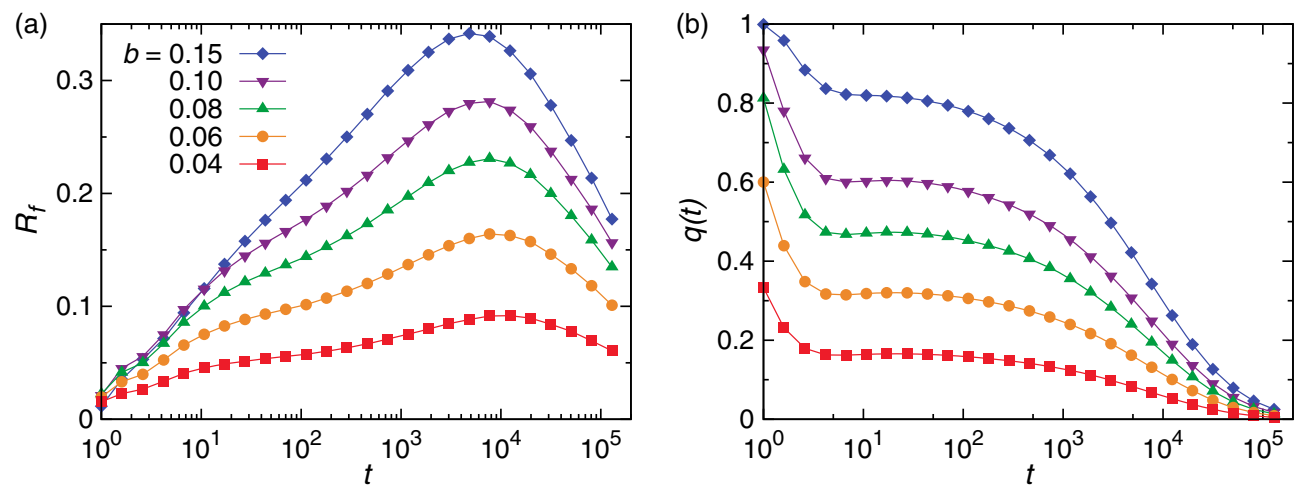

FIG. 10. Signatures of fast $\beta$ dynamics for 2D polydisperse system $(\Delta=11 \%)$ at $T=0.002$. (a) The relative contribution from the structure to the overall dynamic fluctuations $R_{f}$ probed at different cutoff length $b$. (b) Overlap function $q(t)$ according to different cutoff length $b$ for the same system as (a).

Note that the timescales of the initial fast decay and the final decay after the plateau correspond approximately to the shoulder and the peak of $R_{f}$ in Fig. 10(a).

\section{APPENDIX D: FAILURE OF LOCAL POTENTIAL ENERGY AS A STRUCTURAL ORDER PARAMETER}

Here, we examine the importance of many-body correlations in the description of amorphous order in supercooled liquids. In Fig. 11, we show the behavior of local potential energy $E$ upon coarse graining in both polydisperse and binary systems in 2D. The local potential energy of particle $j$ is calculated as $E_{j}=$ $\sum_{k} V\left(r_{j k}\right)$, where the summation goes over all particles that interact with particle $j$. The same as for the other structure order parameters, $E$ is computed in the inherent states to remove the irrelevant random signals. There are conflicting results on whether $E$ can be used as a good structural indicator for dynamics heterogeneity $[28,58,59]$. Since $E$ takes into account only the two-body information (in the case of pair interactions), its failure to describe the subtle amorphous structural order in general can be expected. Indeed, by comparing Fig. 11 with the corresponding parts in Fig. 2, we find that the spatial distribution of $\bar{E}$ appears totally different from that of $\bar{\Psi}_{6}$ and $\bar{\Theta}$ at the same coarse-graining length for polydisperse and binary systems, respectively. Even after coarse graining at the static or dynamic correlation length, the local potential energy shows little correlation with the dynamic propensity at $\tau_{\alpha}$.

We quantify the correlation between structural order based on $\bar{E}$ and the dynamic propensity $\langle\Delta r\rangle$ and show results in Fig. 12. Compared to Figs. 4(a) and 4(d) accordingly, the marginal value of correlation and the absence of a peak correlation at proper time and length scales clearly indicate the failure of $E$. 

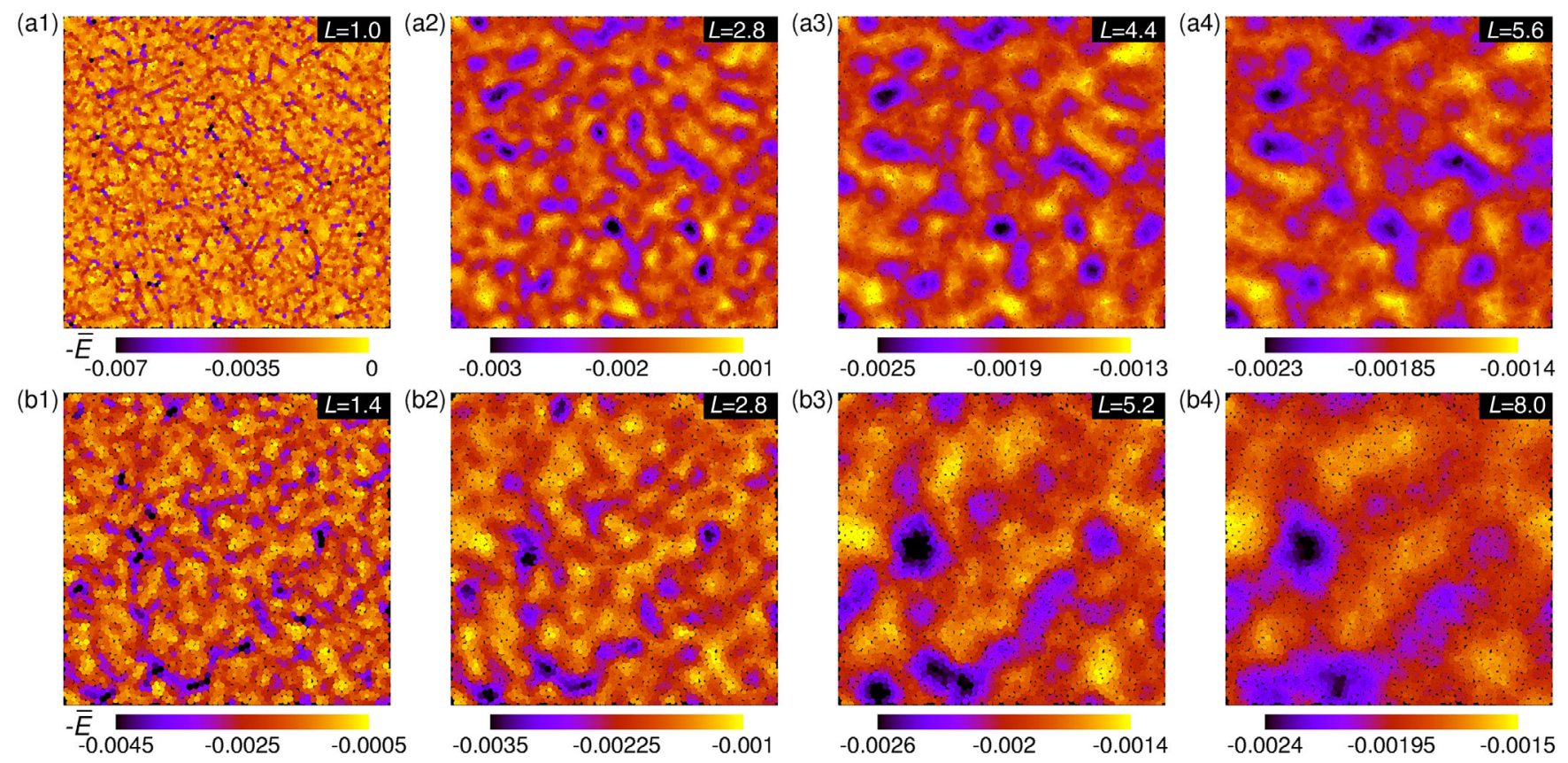

FIG. 11. Coarse graining of local potential energy. (a1)-(a4) Spatial distribution of local potential energy $\bar{E}$ at different coarse-graining length scales $L$ for 2D polydisperse system corresponding to Figs. 2(a) and 2(b). (b1)-(b4) The same as (a) for the 2D binary system corresponding to Figs. 2(c) and 2(d). Note that we have used $-\bar{E}$ in color coding for the ease of comparison.
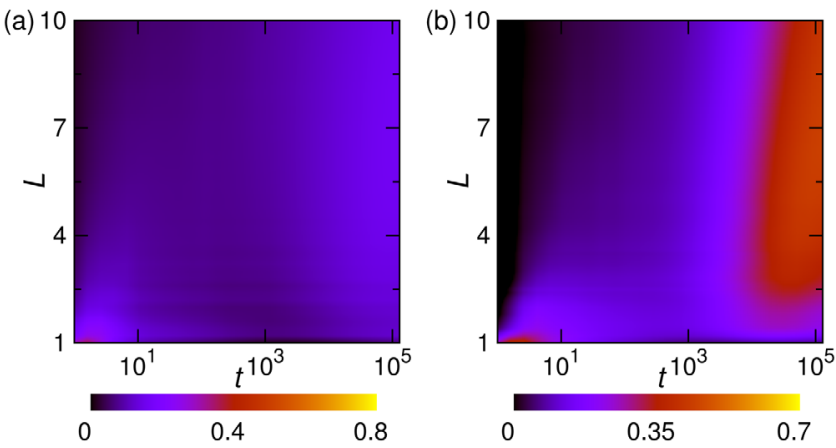

FIG. 12. Cross-correlation of local potential energy and dynamics heterogeneity. Cross-correlation of local potential energy $\bar{E}$ at different coarse-graining length $L$ and the dynamic propensity $\langle\Delta r\rangle$ at different time $t$ for (a) $2 \mathrm{D}$ polydisperse system $(\Delta=11 \%)$ at $T=2 \times 10^{-3}$ and (b) the $2 \mathrm{D}$ binary system at $T=1.5 \times 10^{-3}$. For the ease of comparison, we have used the same color bars as Figs. 4(a) and 4(d) for polydisperse and binary systems, respectively.

Finally, we point out that it is a more serious test of the structure-dynamics correlation by looking at their microscopic correspondence. Previous studies based on the potential energy landscape (PEL) formalism have suggested a clear correlation between the inherent structure energy $E_{\mathrm{IS}}$, that is, the total potential energy of the inherent states (local minima in PEL), and the diffusion coefficient $[59,60]$. It is shown that deeper basins are characterized by sterically favored structures with larger vibrational entropy, and hence, thermodynamically more favored. This is true also in our systems: starting from an initial random state, the inherent structure energy decreases as the system evolves towards equilibrium and the dynamics slows down. However, it does not conflict with our conclusion that a significant microscopic correspondence does not exist between the local potential energy and particle-level dynamics (see Ref. [28] for similar observations in a different system). As a global quantity, $E_{\mathrm{IS}}$ measures the basin depth in PEL, which takes into account all degrees of freedom and therefore contains sufficient many-body information to show correlation with the global dynamics. However, locally, $E$ is too simple to quantify the packing capability and misses the important many-body correlations to correlate with particle-level dynamics. The same is true for the free volume, the global average of which has been applied to understand glassy dynamics [61,62], but fails to explain the dynamic heterogeneity at a local level [32].

[1] C. A. Angell, Formation of Glasses from Liquids and Biopolymers, Science 267, 1924 (1995).

[2] M. D. Ediger, Spatially Heterogeneous Dynamics in Supercooled Liquids, Annu. Rev. Phys. Chem. 51, 99 (2000).

[3] P. G. Debenedetti and F. H. Stillinger, Supercooled Liquids and the Glass Transition, Nature (London) 410, 259 (2001).

[4] J. C. Dyre, Colloquium: The Glass Transition and Elastic Models of Glass-Forming Liquids, Rev. Mod. Phys. 78, 953 (2006). 
[5] L. Berthier and G. Biroli, Theoretical Perspective on the Glass Transition and Amorphous Materials, Rev. Mod. Phys. 83, 587 (2011).

[6] S. Karmakar, C. Dasgupta, and S. Sastry, Growing Length Scales and Their Relation to Timescales in Glass-Forming Liquids, Annu. Rev. Condens. Matter Phys. 5, 255 (2014).

[7] D. Chandler and J. P. Garrahan, Dynamics on the Way to Forming Glass: Bubbles in Space-Time, Annu. Rev. Phys. Chem. 61, 191 (2010).

[8] V. Lubchenko and P. G. Wolynes, Theory of Structural Glasses and Supercooled Liquids, Annu. Rev. Phys. Chem. 58, 235 (2007).

[9] P. M. Chaikin and T. C. Lubensky, Principles of Condensed Matter Physics (Cambridge University Press, Cambridge, England, 2000).

[10] L. Berthier, G. Biroli, J.-P. Bouchaud, L. Cipelletti, and W. van Saarloos, Dynamical Heterogeneities in Glasses, Colloids, and Granular Media, Vol. 150 (Oxford Science Publishers, Oxford, 2011).

[11] R. Yamamoto and A. Onuki, Dynamics of Highly Supercooled Liquids: Heterogeneity, Rheology, and Diffusion, Phys. Rev. E 58, 3515 (1998).

[12] N. Lačević, F. W. Starr, T. B. Schrøder, and S. C. Glotzer, Spatially Heterogeneous Dynamics Investigated via a TimeDependent Four-Point Density Correlation Function, J. Chem. Phys. 119, 7372 (2003).

[13] H. Tanaka, T. Kawasaki, H. Shintani, and K. Watanabe, Critical-like Behaviour of Glass-Forming Liquids, Nat. Mater. 9, 324 (2010).

[14] H. Tanaka, Bond Orientational Order in Liquids: Towards a Unified Description of Water-like Anomalies, Liquid-Liquid Transition, Glass Transition, and Crystallization, Eur. Phys. J. E 35, 113 (2012).

[15] J. S. Langer, Ising Model of a Glass Transition, Phys. Rev. E 88, 012122 (2013).

[16] M. Leocmach, J. Russo, and H. Tanaka, Importance of Many-Body Correlations in Glass Transition: An Example from Polydisperse Hard Spheres, J. Chem. Phys. 138, 12A536 (2013).

[17] J. Russo and H. Tanaka, Assessing the Role of Static Length Scales behind Glassy Dynamics in Polydisperse Hard Disks, Proc. Natl. Acad. Sci. U.S.A. 112, 6920 (2015).

[18] G. M. Hocky, T. E. Markland, and D. R. Reichman, Growing Point-to-Set Length Scale Correlates with Growing Relaxation Times in Model Supercooled Liquids, Phys. Rev. Lett. 108, 225506 (2012).

[19] W. Kob, S. Roldán-Vargas, and L. Berthier, Non-Monotonic Temperature Evolution of Dynamic Correlations in GlassForming Liquids, Nat. Phys. 8, 164 (2012).

[20] B. Charbonneau, P. Charbonneau, and G. Tarjus, Geometrical Frustration and Static Correlations in a Simple Glass Former, Phys. Rev. Lett. 108, 035701 (2012).

[21] S. Karmakar, C. Dasgupta, and S. Sastry, Growing Length and Time Scales in Glass-Forming Liquids, Proc. Natl. Acad. Sci. U.S.A. 106, 3675 (2009).

[22] G. Biroli, S. Karmakar, and I. Procaccia, Comparison of Static Length Scales Characterizing the Glass Transition, Phys. Rev. Lett. 111, 165701 (2013).
[23] I. Tah, S. Sengupta, S. Sastry, C. Dasgupta, and S. Karmakar, Glass Transition in Supercooled Liquids with Medium Range Crystalline Order, arXiv:1705.09532.

[24] C. P. Royall and S. R. Williams, The Role of Local Structure in Dynamical Arrest, Phys. Rep. 560, 1 (2015).

[25] P. Charbonneau and G. Tarjus, Decorrelation of the Static and Dynamic Length Scales in Hard-Sphere Glass Formers, Phys. Rev. E 87, 042305 (2013).

[26] A. Montanari and G. Semerjian, Rigorous Inequalities between Length and Time Scales in Glassy Systems, J. Stat. Phys. 125, 23 (2006).

[27] A. Widmer-Cooper, P. Harrowell, and H. Fynewever, How Reproducible Are Dynamic Heterogeneities in a Supercooled Liquid?, Phys. Rev. Lett. 93, 135701 (2004).

[28] A. Widmer-Cooper and P. Harrowell, Predicting the LongTime Dynamic Heterogeneity in a Supercooled Liquid on the Basis of Short-Time Heterogeneities, Phys. Rev. Lett. 96, 185701 (2006).

[29] L. Berthier and R. L. Jack, Structure and Dynamics of Glass Formers: Predictability at Large Length Scales, Phys. Rev. E 76, 041509 (2007).

[30] A. Widmer-Cooper, H. Perry, P. Harrowell, and D. R. Reichman, Irreversible Reorganization in a Supercooled Liquid Originates from Localized Soft Modes, Nat. Phys. 4, 711 (2008).

[31] S. S. Schoenholz, E. D. Cubuk, D. M. Sussman, E. Kaxiras, and A. J. Liu, A Structural Approach to Relaxation in Glassy Liquids, Nat. Phys. 12, 469 (2016).

[32] A. Widmer-Cooper and P. Harrowell, Free Volume Cannot Explain the Spatial Heterogeneity of Debye-Waller Factors in a Glass-Forming Binary Alloy, J. Non-Cryst. Solids 352, 5098 (2006).

[33] F. C. Frank, Supercooling of Liquids, Proc. R. Soc. A 215, 43 (1952).

[34] P. J. Steinhardt, D. R. Nelson, and M. Ronchetti, BondOrientational Order in Liquids and Glasses, Phys. Rev. B 28, 784 (1983).

[35] G. M. Hocky, D. Coslovich, A. Ikeda, and D. R. Reichman, Correlation of Local Order with Particle Mobility in Supercooled Liquids Is Highly System Dependent, Phys. Rev. Lett. 113, 157801 (2014).

[36] G. Biroli, J. P. Bouchaud, A. Cavagna, T. S. Grigera, and P. Verrocchio, Thermodynamic Signature of Growing Amorphous Order in Glass-Forming Liquids, Nat. Phys. 4, 771 (2008).

[37] S. Yaida, L. Berthier, P. Charbonneau, and G. Tarjus, Pointto-Set Lengths, Local Structure, and Glassiness, Phys. Rev. E 94, 032605 (2016).

[38] B. J. Gellatly and J. L. Finney, Characterisation of Models of Multicomponent Amorphous Metals: The Radical Alternative to the Voronoi Polyhedron, J. Non-Cryst. Solids 50, 313 (1982).

[39] D. R. Nelson, Defects and Geometry in Condensed Matter Physics (Cambridge University Press, Cambridge, England, 2002).

[40] A. Okabe, Spatial Tessellations Concepts and Applications of Voronoi Diagrams (John Wiley \& Sons, Inc., New York, 1992). 
[41] E. Flenner and G. Szamel, Fundamental Differences between Glassy Dynamics in Two and Three Dimensions, Nat. Commun. 6, 7392 (2015).

[42] H. Shiba, Y. Yamada, T. Kawasaki, and K. Kim, Unveiling Dimensionality Dependence of Glassy Dynamics: 2D Infinite Fluctuation Eclipses Inherent Structural Relaxation, Phys. Rev. Lett. 117, 245701 (2016).

[43] B. Illing, S. Fritschi, H. Kaiser, C. L. Klix, G. Maret, and P. Keim, Mermin-Wagner Fluctuations in 2D Amorphous Solids, Proc. Natl. Acad. Sci. U.S.A. 114, 1856 (2017).

[44] S. Vivek, C. P. Kelleher, P. M. Chaikin, and E. R. Weeks, Long-Wavelength Fluctuations and the Glass Transition in Two Dimensions and Three Dimensions, Proc. Natl. Acad. Sci. U.S.A. 114, 1850 (2017).

[45] See Supplemental Material at http://link.aps.org/ supplemental/10.1103/PhysRevX.8.011041 for detailed analyses of glassy dynamics and cross-correlation of structure and dynamics at high temperatures.

[46] E. Bitzek, P. Koskinen, F. Gähler, M. Moseler, and P. Gumbsch, Structural Relaxation Made Simple, Phys. Rev. Lett. 97, 170201 (2006).

[47] M. G. Kendall, Rank Correlation Methods (Griffin, London, 1948).

[48] D. S. Fisher, Activated Dynamic Scaling in Disordered Systems, J. Appl. Phys. 61, 3672 (1987).

[49] T. R. Kirkpatrick, D. Thirumalai, and P. G. Wolynes, Scaling Concepts for the Dynamics of Viscous Liquids Near an Ideal Glassy State, Phys. Rev. A 40, 1045 (1989).

[50] A. S. Keys, L. O. Hedges, J. P. Garrahan, S. C. Glotzer, and D. Chandler, Excitations Are Localized and Relaxation Is Hierarchical in Glass-Forming Liquids, Phys. Rev. X 1, 021013 (2011).

[51] S. Karmakar, C. Dasgupta, and S. Sastry, Short-Time Beta Relaxation in Glass-Forming Liquids Is Cooperative in Nature, Phys. Rev. Lett. 116, 085701 (2016).
[52] J.-P. Hansen and I. R. McDonald, Theory of Simple Liquids (Elsevier, New York, 1990).

[53] W. Gotze and L. Sjogren, Relaxation Processes in Supercooled Liquids, Rep. Prog. Phys. 55, 241 (1992).

[54] E. R. Weeks, J. C. Crocker, A. C. Levitt, A. Schofield, and D. A. Weitz, Three-Dimensional Direct Imaging of Structural Relaxation Near the Colloidal Glass Transition, Science 287, 627 (2000).

[55] W. K. Kegel and A. van Blaaderen, Direct Observation of Dynamical Heterogeneities in Colloidal Hard-Sphere Suspensions, Science 287, 290 (2000).

[56] M. Leocmach and H. Tanaka, Roles of Icosahedral and Crystal-like Order in the Hard Spheres Glass Transition, Nat. Commun. 3, 974 (2012).

[57] P. J. Yunker, K. Chen, M. D. Gratale, M. A. Lohr, T. Still, and A.G. Yodh, Physics in Ordered and Disordered Colloidal Matter Composed of Poly (N-Isopropylacrylamide) Microgel Particles, Rep. Prog. Phys. 77, 056601 (2014).

[58] G. S. Matharoo, M. S. Gulam Razul, and P. H. Poole, Structural and Dynamical Heterogeneity in a Glass-Forming Liquid, Phys. Rev. E 74, 050502 (2006).

[59] E. La Nave, S. Sastry, and F. Sciortino, Relation between Local Diffusivity and Local Inherent Structures in the KobAndersen Lennard-Jones Model, Phys. Rev. E 74, 050501 (2006).

[60] F. Sciortino, Potential Energy Landscape Description of Supercooled Liquids and Glasses, J. Stat. Mech. (2005) $\mathrm{P} 05015$.

[61] M. H. Cohen and G. S. Grest, Liquid-Glass Transition, A Free-Volume Approach, Phys. Rev. B 20, 1077 (1979).

[62] F. W. Starr, S. Sastry, J. F. Douglas, and S. C. Glotzer, What Do We Learn from the Local Geometry of Glass-Forming Liquids?, Phys. Rev. Lett. 89, 125501 (2002). 